\title{
Beam Dynamics Simulations for the Low-Energy Magnetic Bunch Compressor at ASTA
}

\author{
C. R. Prokop, ${ }^{1}$ P. Piot, ${ }^{1,2}$ and M. Church $^{3}$ \\ ${ }^{1}$ Northern Illinois Center for Accelerator $\&$ \\ Detector Development and Department of Physics, \\ Northern Illinois University, DeKalb IL 60115, USA \\ ${ }^{2}$ Accelerator Physics Center, Fermi National \\ Accelerator Laboratory, Batavia, IL 60510, USA \\ ${ }^{3}$ Accelerator Division, Fermi National Accelerator Laboratory, Batavia, IL 60510, USA
}

(Dated: December 5, 2012)

\begin{abstract}
The Advanced Superconducting Test Accelerator (ASTA) at Fermilab incorporates a magnetic chicane bunch compressor to enhance the electron-bunch peak current. The bunch compression occurs at an energy of $40 \mathrm{MeV}$ and is therefore subject to significant phase-space degradations which occur as a result of space-charge and coherent synchrotron radiation effects. In this report, we explore the performance of this low-energy bunch compressor using several simulation programs.
\end{abstract}




\section{INTRODUCTION}

The Advanced Superconducting Test Accelerator (ASTA) at Fermilab is a superconducting linac currently in construction at FNAL's New Muon Laboratory. ASTA will eventually generate electron beams suitable to support a variety of user-driven experiments in advanced accelerator R\&D (AARD) [1,2]. The facility's construction is staged and will initially be composed of a $\sim 40 \mathrm{MeV}$ photoinjector followed by one accelerating cryomodule [3]; see Fig. 1. This setup is henceforth referred to as "first-beam" configuration. Compressed bunches with sub-ps duration will be highly beneficial for AARD experiments and also enable investigation of topics relevant to the International Linear Collider (ILC) program such as, e.g., long-range wakefield in superconducting cavities. Therefore a magnetic bunch compressor (BC1 in Fig. 1) is incorporated in the injector area downstream of the accelerating section at $40 \mathrm{MeV}$. The location and design of BC1 was essentially dictated by space constraints and is not optimal: compressing the bunch at such low energies will result in detrimental phase-space dilutions due to collective effects such as coherent synchrotron radiation (CSR) and space charge (SC). BC1 will eventually be the first compressor of a two-stage compression scheme and will only provide mild compression. For first beam operation, BC1 will fully compress the bunch, thereby enabling the production of high-peak current $(\sim 5 \mathrm{kA})$ electron bunches.

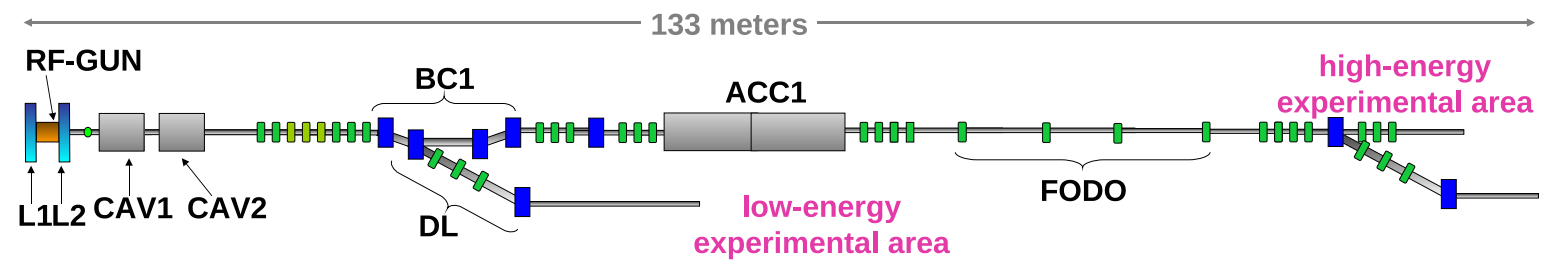

FIG. 1: Overview of the "first-beam" beamline configuration for ASTA. "RF-Gun" is the photocathode gun, "L1" and "L2" are solenoid magnets, "CAV1" and "CAV2" are nine-cell superconducting RF accelerating cavities, "BC1" is the low-energy bunch compressor, "DL" is a dogleg to the low-energy experimental area, "ACC1" is the first eight-cavity cryomodule, and "FODO" is the FODO lattice.

In this report, we explore the performance of the BC1 low-energy bunch compressor for various bunch charges. We especially investigate a possible trade-off between high-peak current and transverse-emittance preservation. Our numerical studies are performed with 
several models to account for CSR and/or SC available in popular beam dynamics programs. The programs used for our simulations are, when possible, benchmarked against each other.

\section{BUNCH COMPRESSOR DESIGN AND THEORY}

The photoinjector beamline configuration considered in this report is diagrammed in Fig. 2. In the photoinjector, the beam is generated from a photoemission electron source (the "RF gun") and accelerated to $\sim 40 \mathrm{MeV}$ in two superconducting cavities (CAV1 and CAV2). In this process, the operating parameters are tuned to minimize the transverse emittance [4]. In order to generate a low transverse emittance, the bunch-charge density is reduced by illuminating the photocathode with a long laser pulse (initially using a single 3ps ultraviolet pulse and later using a 10- to 20-ps flat-top pulse obtained by stacking several 3-ps pulses). The produced long (duration of several ps) electron bunch results in significant longitudinal emittance growth as the beam is accelerated to its final energy. This growth stems from the quadratic correlations imposed by the rf-wave curvature in the longitudinal phase space (LPS). These correlations can be removed with a third-order accelerating cavity (CAV39) [5-8]. This cavity will not be available for first-beam operation.

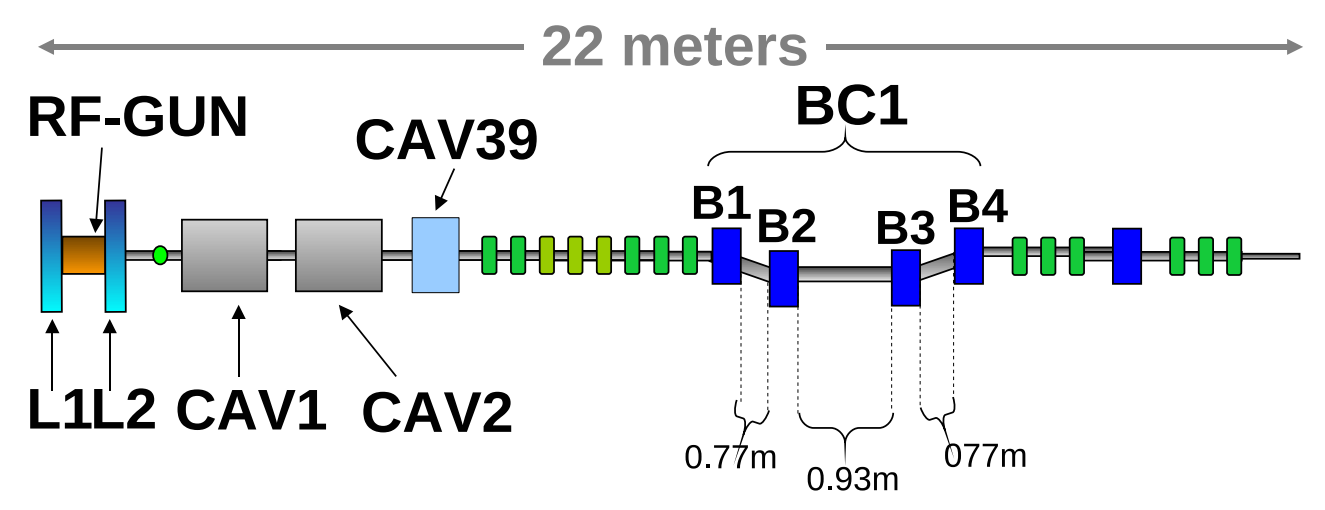

FIG. 2: Overview of the ASTA photoinjector. The "RF-gun", "L1" and "L2" respectively correspond to the gun cavity and surrounding solenoid magnets, "CAV1", "CAV2", and "CAV39" are superconducting rf cavities, "BC1" refers to the magnetic bunch compressor, and B1-4 are the dipoles of the chicane, with distance between the dipoles marked in the figure.

Prior to injection in the cryomodule, the bunch can be longitudinally compressed using the magnetic bunch compressor (BC1) which, consists of four 0.2-m rectangular dipoles 
(B1, B2, B3, B4) with bending angles of $(+,-,-,+) 18^{\circ}$; see Fig. 2 for distances between the dipoles. This study is based on an early design for the low-energy bunch compressor; the chicane's dipole lengths and drift distances may change for the final experiment, and the bunch energy in the injector may be increased up to $\sim 50 \mathrm{MeV}$. In this process, an electron has its initial LPS coordinate $\left(z_{0}, \delta_{0}\right)$ upstream of BC1 mapped to the final LPS coordinates downstream of $\mathrm{BC} 1$

$$
\left\{\begin{array}{l}
z_{f}=z_{0}+R_{56} \delta_{0} \\
\delta_{f}=\delta_{0}
\end{array}\right.
$$

where $R_{56}$ is the longitudinal dispersion associated with BC1 taken to be negative for a chicane-type compressor. Here we neglect possible collective effects and only considered the first-order transfer map. The final root-mean-square (RMS) bunch length is

$$
\begin{aligned}
\sigma_{z, f} & =\left[\sigma_{z, 0}^{2}+R_{56}\left\langle z_{0} \delta_{0}\right\rangle+R_{56}^{2} \sigma_{\delta, 0 u}^{2}\right]^{1 / 2} \\
& =\sigma_{z, 0}\left[1+R_{56} \mathcal{C}+R_{56}^{2} \frac{\sigma_{\delta, 0 u}^{2}}{\sigma_{z, 0}^{2}}\right]^{1 / 2}
\end{aligned}
$$

Therefore, with appropriate initial LPS chirp, $\mathcal{C} \equiv-\left\langle z_{0} \delta_{0}\right\rangle / \sigma_{z, 0}^{2}$, the bunch compression can be varied. When $\mathcal{C}=-1 / R_{56}$ the bunch length reach its minimal value limited by the uncorrelated fractional momentum spread $\sigma_{\delta, 0 u}$. The $R_{56}$ of the chicane is $19 \mathrm{~cm}$, which indicates that the greatest compression for a perfectly linearized beam will occur for $\mathcal{C} \approx 5.25 \mathrm{~m}^{-1}$. We operate CAV1 on-crest, and use only-CAV2 to impart the energy-chirp, to avoid velocity bunching and its complications. Therefore a simple model of the longitudinal dynamics can be developed.

Consider an electron emitted by the rf gun with energy and position related by

$$
E(z)=E_{g}+a z+b z^{2}
$$

where $E_{g}, a$, and $b$ are parameters that depend on the rf gun operating parameters. Taking the average beam energy to be $E_{g}$ downstream of the gun and considering the acceleration voltage through CAV1 and CAV2 to be

$$
V(z)=V_{1} \cos \left(k z+\varphi_{1}\right)+V_{2} \cos \left(k z+\varphi_{2}\right)
$$


and expanding in $z$, we have (to second order) the energy gain from CAV1 and CAV2

$$
\begin{aligned}
\Delta E_{1+2}(z)= & e V_{1} \cos \left(\varphi_{1}\right)+e V_{2} \cos \left(\varphi_{2}\right) \\
& -e V_{1} k z \sin \left(\varphi_{1}\right)-e V_{2} k z \sin \left(\varphi_{2}\right) \\
& -\frac{1}{2} e V_{1} k^{2} z^{2} \cos \left(\varphi_{1}\right)-\frac{1}{2} e V_{2} k^{2} z^{2} \cos \left(\varphi_{2}\right) .
\end{aligned}
$$

The total energy downstream of CAV2 is then

$$
\begin{aligned}
E(z)= & E_{g}+a z+b z^{2}+V_{1} \cos \left(\varphi_{1}\right)+V_{2} \cos \left(\varphi_{2}\right) \\
& -V_{1} k z \sin \left(\varphi_{1}\right)-V_{2} k z \sin \left(\varphi_{2}\right) \\
& -\frac{1}{2} e V_{1} k^{2} z^{2} \cos \left(\varphi_{1}\right)-\frac{1}{2} e V_{2} k^{2} z^{2} \cos \left(\varphi_{2}\right) .
\end{aligned}
$$

Linearization of the LPS requires that the sum of the $z^{2}$ terms goes to zero, which we accomplish with a third harmonic cavity, CAV39, operating at $3.9 \mathrm{GHz}$, which can be similarly expanded as

$$
\Delta E_{39}(z)=e V_{39} \cos \left(\varphi_{39}\right)-e V_{39} k z \sin \left(\varphi_{39}\right)-\frac{1}{2} e V_{39} k_{39}^{2} z^{2} \cos \left(\varphi_{39}\right)
$$

If we are to use CAV39 for linearization, we then have the requirement

$$
b-\frac{1}{2} e V_{1} k^{2} \cos \left(\varphi_{1}\right)-\frac{1}{2} e V_{2} k^{2} \cos \left(\varphi_{2}\right)-\frac{1}{2} e V_{39} k_{39}^{2} \cos \left(\varphi_{39}\right)=0 .
$$

which can be rearranged

$$
V_{39} \cos \left(\varphi_{39}\right)=\frac{1}{9}\left[\frac{2 b}{e k^{2}}-V_{1} \cos \left(\varphi_{1}\right)-V_{2} \cos \left(\varphi_{2}\right)\right] .
$$

In addition to the linearization, we have a second condition imposed by our requirement of maximum compression, so that the terms linear in $z$, i.e. the LPS chirp, is related to the $R_{56}$ of the chicane:

$$
\frac{a-e V_{1} k \sin \left(\varphi_{1}\right)-e V_{2} k \sin \left(\varphi_{1}\right)-e V_{39} 3 k \sin \left(\varphi_{39}\right)}{E_{g}+e V_{1} \cos \left(\varphi_{1}\right)+e V_{2} \cos \left(\varphi_{2}\right)+e V_{39} \cos \left(\varphi_{39}\right)}=-\frac{1}{R_{56}} .
$$

Substituting Eq. 8 into the denominator gives

$$
\frac{a-e V_{1} k \sin \left(\varphi_{1}\right)-e V_{2} k \sin \left(\varphi_{2}\right)-e V_{39} 3 k \sin \left(\varphi_{39}\right)}{E_{g}+\frac{8}{9} e V_{1} \cos \left(\varphi_{1}\right)+\frac{8}{9} e V_{2} \cos \left(\varphi_{2}\right)+\frac{2 b}{9 k^{2}}}=-\frac{1}{R_{56}} .
$$

Rearranging to isolate $V_{39} \sin \left(\varphi_{39}\right)$, we find

$$
\begin{gathered}
V_{39} \sin \left(\varphi_{39}\right)=\frac{1}{3 k R_{56}}\left[E_{g}+\frac{8}{9} e V_{1} \cos \left(\varphi_{1}\right)+\frac{8}{9} e V_{2} \cos \left(\varphi_{2}\right)+\frac{2 b}{9 k^{2}}\right] \\
+\frac{1}{3 k} V_{1} \cos \left(\varphi_{1}\right)+\frac{1}{3 k} V_{2} \cos \left(\varphi_{2}\right)-\frac{1}{3 k} a
\end{gathered}
$$


Combining Eqs. 8 and 12, we obtain the expression for the operating phase,

$$
\varphi_{39}=\tan ^{-1}\left(\frac{\frac{1}{3 k R_{56}}\left[E_{g}+\frac{8}{9} e V_{1} \cos \left(\varphi_{1}\right)+\frac{8}{9} e V_{2} \cos \left(\varphi_{2}\right)+\frac{2 b}{9 k^{2}}\right]+\frac{1}{3 k} V_{1} \cos \left(\varphi_{1}\right)+\frac{1}{3 k} V_{2} \cos \left(\varphi_{2}\right)-\frac{1}{3 k} a}{\frac{1}{9}\left[\frac{2 b}{e k^{2}}-V_{1} \cos \left(\varphi_{1}\right)-V_{2} \cos \left(\varphi_{2}\right)\right]}\right)
$$

The recovered operating phase can then be substituted back into Eq. 8 to find the voltage.

We now consider our nominal operating condition where CAV1 is on-crest $\left(\varphi_{1}=0\right)$ and CAV2's phase $\varphi_{2}$ is used to impart the LPS chirp. Typical operating parameters for the ASTA photoinjector are $E_{g}=5 \mathrm{MV}, V_{1}=12 \mathrm{MV}$, and $V_{2}=22 \mathrm{MV}$, and we take $\varphi_{2}=-27^{\circ}$. For simplicity, we ignore initial LPS linear and quadratic terms arising from the rf gun $(a=b=0)$. Following the previous equations, the phase and voltage for CAV39 are then $\varphi_{39}=170^{\circ}$ and $V_{39}=3.6 \mathrm{MV}$.

Since we will not always be phasing the cavities to achieve maximum compression, we show in Fig. 3 the operating phase $\phi_{39}$ and voltage $V_{39}$ for CAV39 to linearizie the LPS as functions of the phase of CAV2.
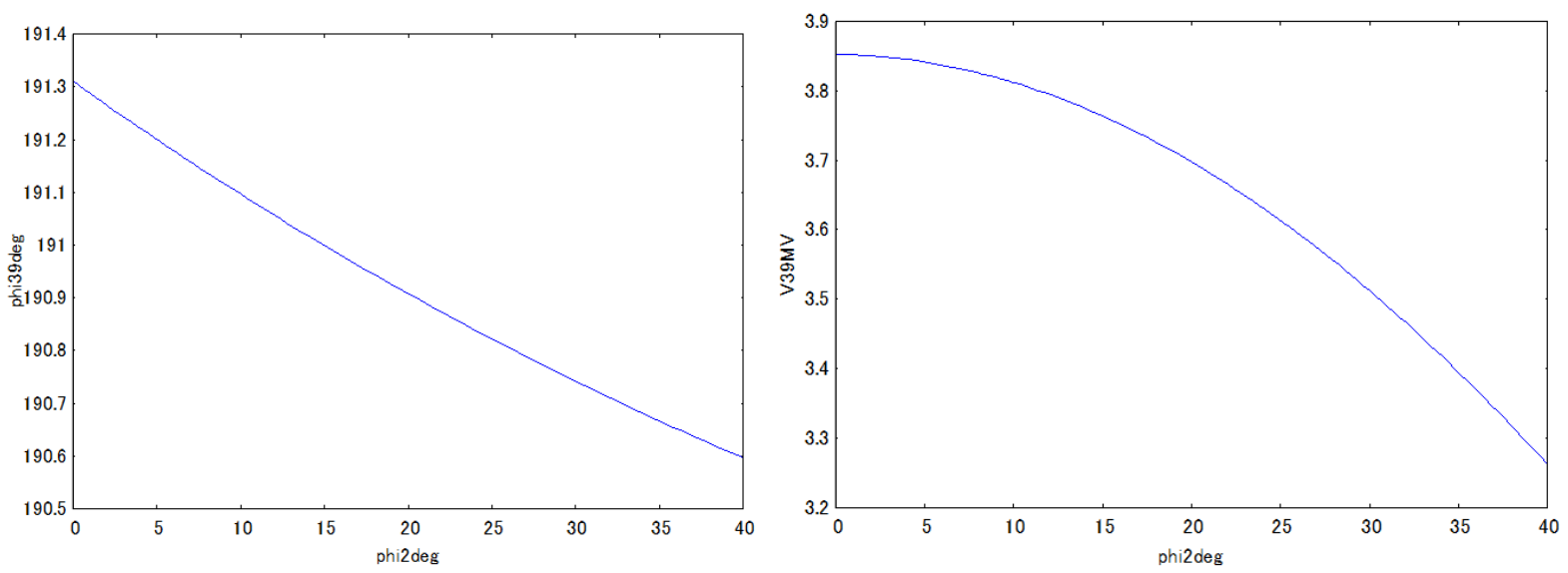

FIG. 3: The operating phase $\phi_{39}$ (left) and voltage $V_{39}$ (right) for CAV39 as functions of the operating phase of CAV2, $\phi_{2}$, using its voltage $V_{2}=22 \mathrm{MV}$.

When collective effects are taken into account the beam dynamics can significantly deviate from the simple model just discussed. In the case of BC1 in particular, because the electrons travel on a curved trajectory while within the field of a dipole magnet, they emit synchrotron radiation. This radiation leads to a net energy loss (which is insignificant in the case of $\mathrm{BC} 1$ ) but can also feedback on the beam and introduce an energy redistribution. For nanocoulomb bunch charges circulating on trajectories with short bending-radii, CSR emitted from the tail of the bunch overtakes the head of the bunch, imparting an energy modulation along the 
bunch's length. Since this energy modulation occurs within a dispersive location, it locally "breaks" the achromaticity of the chicane and results in a transverse emittance growth. A one-dimensional model of the corresponding energy redistribution along a bunch traveling on a curved trajectory is detailed in Ref. [9]. This model serves as the basis for the CSR models implemented in several of the simulation codes discussed here. The energy redistribution associated with the CSR longitudinal force is

$$
\left(\frac{\partial E}{\partial s}\right)_{C S R}=\frac{2 Q}{R^{2 / 3} 3^{1 / 3}} \int_{-\infty}^{z} \frac{1}{\left(z-z^{\prime}\right)^{1 / 3}} \frac{\partial \lambda\left(z^{\prime}\right)}{\partial z^{\prime}} d z^{\prime}
$$

where $Q$ is the bunch charge, $R$ is the bend radius, $\lambda(z)$ is the longitudinal projection of the charge distribution, $z$ is the position of the source particle, and $z^{\prime}$ is that of the witness particle. For a sufficiently long dipole and Gaussian charge distribution, this approximation is characterized by an energy gain near the head of the bunch and an average fractional energy loss of [10]

$$
\Delta \delta=-3.505 \frac{r_{e} Q L_{b}}{e \gamma \sigma_{z}^{4 / 3} R^{2 / 3}}
$$

where $r_{e}$ is the classical electron radius, $Q$ is bunch charge, $e$ is the election charge, and $L_{b}$ is the path length through the dipole. This uncorrelated energy-spread leads to a growth in the transverse emittance due to the change in particle energy mid-chicane.

This semi-analytical approach to CSR assumes the electron bunch as a line charge distribution so that the transverse dimensions of the beam are ignored. The validity of this model is quantified by the Derbenev's criterion

$$
\frac{\sigma_{x}}{\sigma_{z}} 2^{1 / 3}\left[3 \frac{R^{2}}{\sigma_{z}^{2}}\right]^{1 / 9} \ll 1,
$$

where $\sigma_{x}$ and $\sigma_{z}$ are the transverse and longitudinal bunch sizes, and $R$ is the bending radius of the dipole, which for our dipoles is $0.65 \mathrm{~m}$. The parameters represented in the criterion vary along the length of the chicane and depends on which bunch charge we are using, but at the fourth dipole (where CSR is expected to be greatest), $\sigma_{z}$ is on the order of 100 s of $\mu \mathrm{m}$, and the Derbenev's criterion is generally not met. Therefore simulations based on the one-dimensional model should be considered with care.

Space-charge forces are intricate to consider as the are fully tree-dimensional. However, longitudinal space charge (LSC) can be readily compared with CSR as it introduces an 
energy loss given by $[16]$

$$
\left(\frac{\partial E}{\partial s}\right)_{L S C}=-\frac{2 e}{\gamma^{2}} \frac{\partial \lambda(z)}{\partial z}
$$

where $e$ is the election charge.

The characteristic forces of LSC in a drift and CSR in a dipole are shown in Fig. 4. Although both of these effects predominantly affect the longitudinal phase spaces, they can couple to the transverse phase space when occurring, for example, in a dispersive section. In addition, the transverse phase space is also altered by transverse space charge forces. The key difference between the energy losses is the lack of energy-dependence in the simple 1-D CSR model.

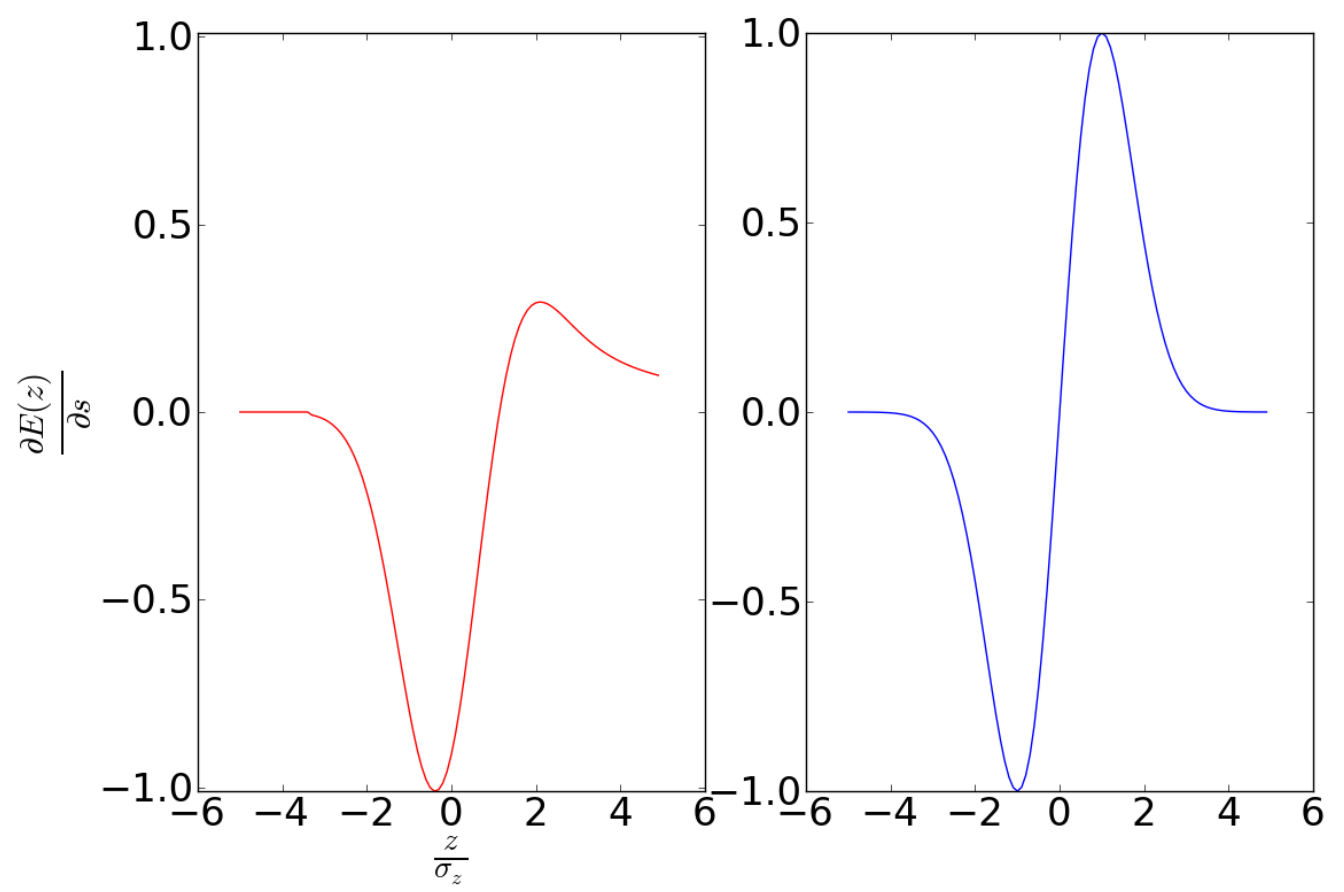

FIG. 4: Characteristic forces of CSR in a dipole (left) and LSC in a drift (right). The CSR force is normalized to $\frac{23 Q}{\sigma_{z} R^{2 / 3} 3^{1 / 3} \sqrt{(2 \pi)}}$, and the $\mathrm{SC}$ force is normalized to $\frac{2 Q e^{-1 / 2}}{\sigma_{z} \gamma^{2}}$.

\section{SIMULATION CODE OVERVIEW}

The simulation of collective effects along the photoinjector was, in our previous work [11], executed with ELEGant[12] and ImpaCt-Z[13]. In this paper, we concentrate on BC1, 
and use the two codes used in our previous work, as well as the CSR-focused software CSRTRACK[14].

Due to the different conventions used in each of the different simulation codes, we use a consistent standard throughout this paper. We use the convention that the head of the bunch corresponds to $z>0$, the transverse dimension $x$ is the chicane's bending plane, and $y$ is the vertical transverse direction. The fractional energy deviation relative to the reference particle is defined as $\delta \equiv \frac{E}{\langle E\rangle}-1$, and a positive LPS chirp corresponds to the tail of the bunch being a higher energy than its head.

Due to the range of energies along the ASTA beamline and the inadequacy of any single simulation code to accurately model all the collective effects of the electron bunches, we use a sequence of simulation codes to perform start-to-end (S2E) simulations. We use AstrA to model the photocathode gun and first-stage acceleration to $\sim 40 \mathrm{MeV}$ (through CAV2 in Fig. 2), and subsequently ElEGAnt, ImpaCT-Z, or CSRTRACK are used depending on the desired study.

ELEGANT is a 6-D tracking code capable of tracking a variety of different particle types through both linear and circular accelerators. While it allows for some simple models of collective effects, here we use it only for beamline design and optimization, as well as secondorder single-particle tracking.

IMPACT-Z includes a quasi-static 3-D space charge (SC) particle-in-cell (PIC) algorithm and a 1-D model of coherent synchrotron radiation (CSR). The CSR-induced energy loss is computed from the longitudinal charge distribution obtained from a longitudinal binning of the macroparticle distribution using the formalism of Saldin. In this paper, we utilize IMPACT-Z in two different configurations; one, where both collective effects are utilized in the bunch compressor (SC+CSR), and a second one (SC-only) where we disable the CSR calculation to assess only the effects of SC. Like ELEGANT, the IMPACT-Z includes fringe fields using the FINT parameter, which adjusts the transverse focusing at the dipole edges.

CSRTRACK is a simulation developed by Dohlus, et al., that is specifically designed to utilize numerous models in simulating CSR forces along circular trajectories in magnetic fields. One is a 2-D "Particle to Particle" (P2P) model that solves the Lienard-Wiechart potentials at retarded times, calculating both the transverse and longitudinal contributions from SC and CSR. Since the P2P model is computationally intensive (the calculation time scales as $N^{2}$ where $N$ is the number of macroparticles), CSRTRACK also has an improved 
1-D model referred to as the 1D-Projected (1DP) model, which uses the 1-D projection of the smoothed charge distribution convoluted with a kernel function $K\left(z_{0}, s\right)$ [15],

$$
E\left(u_{o}\right)=\int \lambda^{\prime} K\left(s_{o}, s\right) d s
$$

where the kernel is given by

$$
K\left(s_{o}, s\right)=\frac{\beta \bar{n} \cdot\left(\vec{u}_{s}-\vec{u}_{o}\right)-\beta^{2}\left(1-\vec{u}_{s} \cdot \vec{u}_{o}\right)-\gamma^{-2}}{R}+\frac{1-\beta \vec{u}_{s} \cdot \vec{n}}{\gamma^{2}|s+\beta R|} .
$$

In these equations $s$ is the source particle position, $s_{o}$ is the observer particle position, $\overrightarrow{u_{s}}$ and $\overrightarrow{u_{o}}$ are the position vector of the source and observer particles, respectively, $\vec{n}=\frac{\vec{R}}{|\vec{R}|}$, $R=|\vec{R}|, \beta=\frac{v}{c}$, and $\lambda$ is the longitudinal projection.

Both models impose finite sizes to the macroparticles as gaussian sub-bunches in order to smooth the distributions and make them less prone to numerical noise from having a limited number of macroparticles, as is necessary when performing computationally intensive P2P simulations. The current distribution $\lambda(z)$ is the sum of the projection of each macroparticle, or

$$
\lambda(z)=\frac{1}{\sigma_{B} \sqrt{2 \pi}} \sum_{i=1}^{N} q_{i} e^{\left(\frac{-\left(z-z_{i}\right)^{2}}{2 \sigma_{B}^{2}}\right)}
$$

where $\sigma_{B}$ is the RMS of the gaussian sub-bunch. For fully 3D bunches, $\sigma_{B}$ is set separately for each dimension, and the smoothed distribution is of the form

$$
F(x, y, z) \propto \frac{1}{\sigma_{B_{x}} \sigma_{B_{y}} \sigma_{B_{z}}(2 \pi)^{3 / 2}} \sum_{i=1}^{N} q_{i} e^{\left(\frac{-\left(x-x_{i}\right)^{2}}{2 \sigma_{B x}^{2}}\right)} e^{\left(\frac{-\left(y-y_{i}\right)^{2}}{2 \sigma_{B y}^{2}}\right)} e^{\left(\frac{-\left(z-z_{i}\right)^{2}}{2 \sigma_{B z}^{2}}\right)}
$$

CSRTRACK includes a fringe field kick, but it is not a parameter that can be controlled by the user. The longitudinal dynamics in the direction of the bend plane, $x$, match to around $5 \%$ of the IMPACT-Z simulations, while the vertical dynamics (which play no role in CSRTRACK's calculations), are over-focused compared to the IMPACT-Z simulations, and are off by considerably more than the $x$ dynamics.

Note that the simulations presented in this paper account for only the short distance of one meter after the 4 th dipole of the chicane, while space charge effects (and some tail of 
CSR that travels along with the bunch after the dipole) are of continuing detriment to the phase space until it is accelerated in the first cryomodule, which reduces the relative effects of SC.

\section{BUNCH COMPRESSOR BEAM OPTICS}

The code ELEGANT was used to model and optimize the single-particle dynamics throughout the ASTA beamline. Two examples of LPS distributions tracked through BC1 for an ideal compression, i.e. in absence of collective effects, appear in Fig. 5. The simulations, carried out with ELEGANT, illustrate the benefits of the LPS linearization using CAV39 toward significantly improving the peak current. For these simulations, the LPS is modeled upstream of CAV2 with ASTRA as a 3.2-nC bunch, which is then loaded into ELEGANT with matching functions performed via GLUETRACK, with and without the linearization process detailed earlier. The linearization of the LPS produced by a 3.9-GHz cavity is modeled with the transformation $\delta \rightarrow \delta_{0}-b z_{0}^{2}$ via a set of PYTHON scripts called GLUETRACK, where the parameter $b$ is obtained from a polynomial fit of the incoming LPS distribution $\left(z_{0}, \delta_{0}\right)$. GlueTRACK is also used to match the Courant-Snyder (C-S) parameters and energies of the distributions, and to adjust the energy chirp of the bunch, as the original AsTRA simulations which were used to generate the different distributions were not optimized to produce distributions of equal energy or C-S parameters, and because re-running the AsTRA simulations to generate new distributions would be time-prohibitive.

Without collective effects, the peak currents for a linearized $3.2 \mathrm{nC}$ bunch may exceed $12 \mathrm{kA}$, and is limited by the uncorrelated energy spread $\hat{I} \simeq \frac{Q c}{R_{56} \sigma_{\delta u} \sqrt{2 \pi}}$, where $\sigma_{\delta u}$ is the uncorrelated fractional momentum spread.

To determine the optimal C-S parameters for the minimization of CSR effects, we performed a scan of initial C-S parameters using CSRTRACK's 1DP model (shown in Fig. 6) to find the operating regimes of least emittance-growth. The "valley" of minimum growth in the lower-right hand corner of the plot coincides with the C-S parameters that lead to a waist between the third and fourth dipole, at which its projected slice length is greatest [17]. The upper "valley" corresponds to a waist between the 1st and 2nd dipoles, though Fig. 7 shows that such a solution may lead to large-valued betatron functions at the chicane exit which would complicate the beam optics downstream of the chicane and render the lattice 

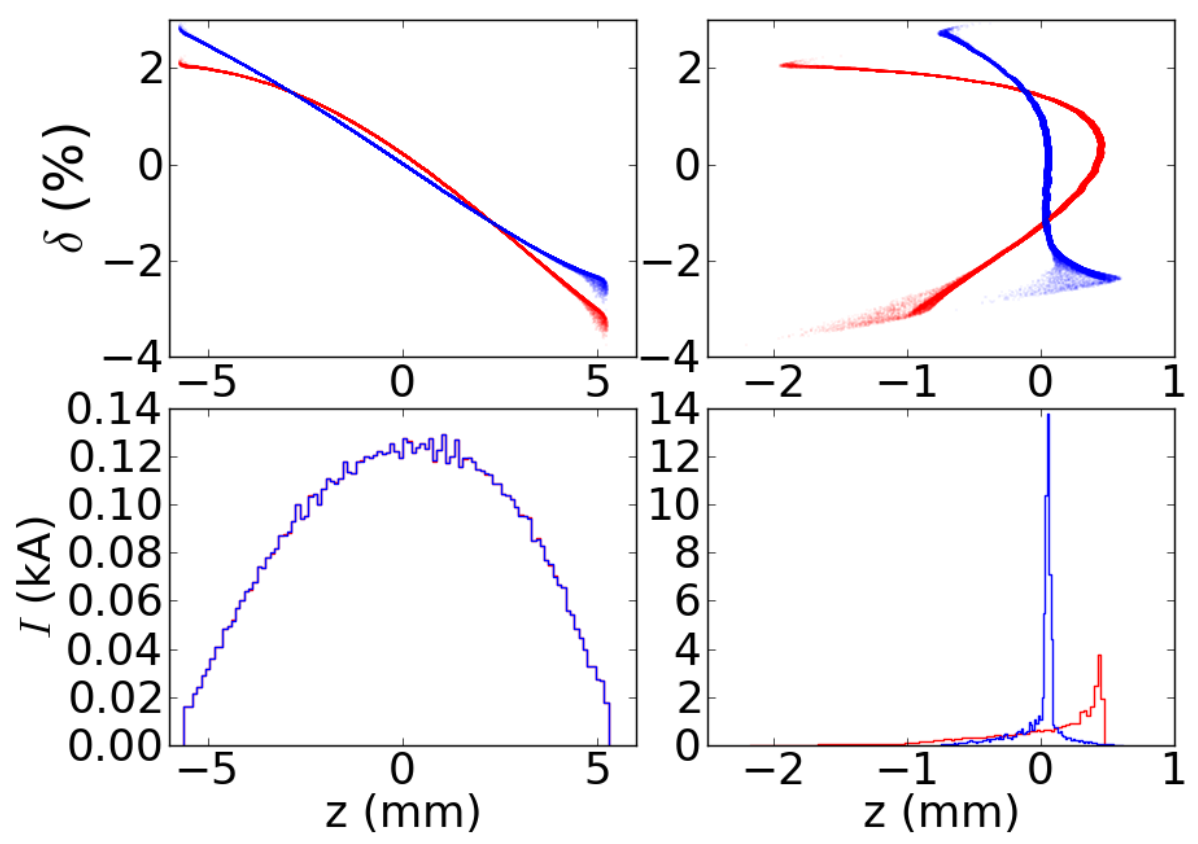

FIG. 5: LPS distributions (top row) and associated current profiles (bottom row) before (left column) and after (right column) BC1, without collective effects. The red and blue traces correspond respectively to the linearized and nominal initial LPS of a 3.2-nC bunch, showing the vastly higher peak currents that become available by using the third-harmonic cavity for LPS linearization.

more prone to aberrations.

Using the results of the C-S scan, we determined the baseline operating parameters for the low-energy chicane (see Table I. These CSRTRACK simulations give us the case of $\alpha_{x}=3$ and $\beta_{x}=8$ at $0.1 \mathrm{~m}$ upstream of the first dipole, which leads to a waist in $\mathrm{x}$ between the third and fourth dipoles and recoverable C-S parameters at the exit of BC1. For the other transverse dimension, we chose $\alpha_{y}=-1.6$ and $\beta_{y}=1.6 \mathrm{~m}$, which gives a betatron function that is controlled and easily achievable, and has outgoing lattice functions that are easy to match to.

\section{IMPACT-Z PARAMETRIC STUDIES}

IMPACT-Z has several simulation parameters that influence the computational intensity and physical accuracy of the simulations, including grid size for the SC and CSR calculations and the number of macroparticles used in each simulation. We needed to determine what 

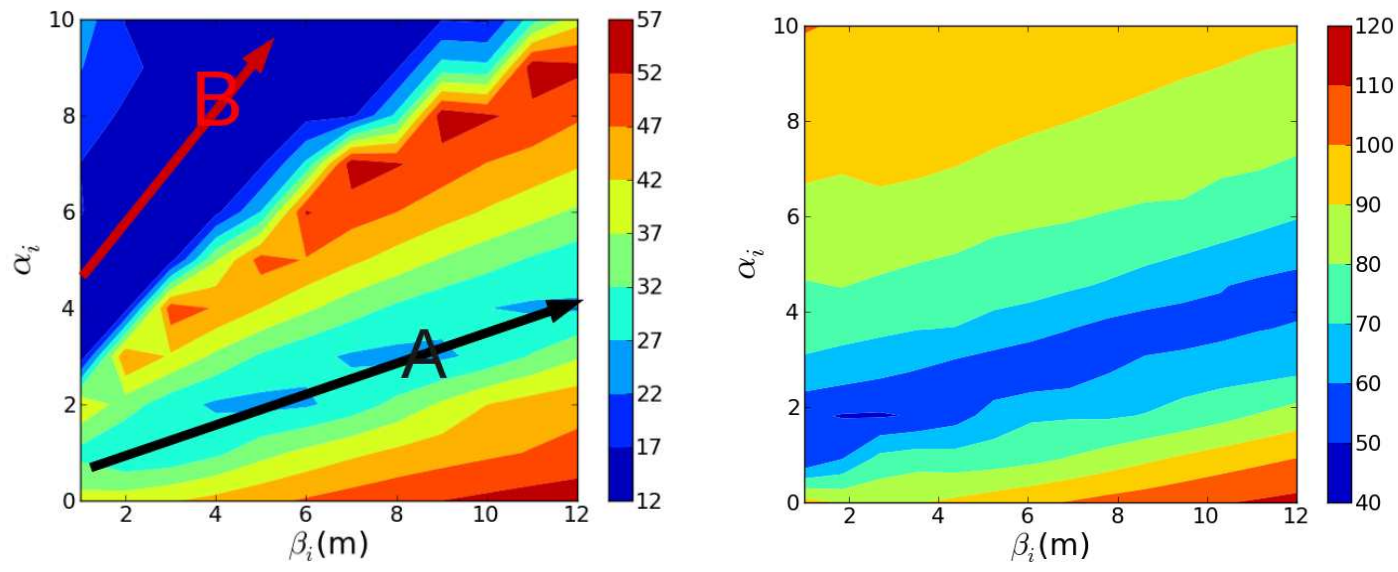

FIG. 6: Contour plot of the final normalized horizontal emittance $\left(\varepsilon_{n x}\right.$ in $\left.\mu \mathrm{m}\right)$ a function of the initial C-S parameters $\beta_{i}$ and $\alpha_{i}$ for the nominal (left) and linearized (right) incoming LPS. The simulations are performed with CSRTRACK's 1D-Projected model. The black arrow indicates a region where the beam reaches a waist in $\mathrm{x}$ between the 3rd and 4th dipoles, and the red arrow indicates where the beam reaches a waist beween the 1st and 2nd dipoles. The label "A" indicates the operating point for most of the simulations performed in this report.

TABLE I: Baseline operating parameters for the low-energy chicane.

\begin{tabular}{ccc}
\hline Parameter & Value & Units \\
\hline \hline$\beta_{x_{i}}$ & 8 & $\mathrm{~m}$ \\
$\alpha_{x_{i}}$ & 3 & - \\
$\beta_{y_{i}}$ & 1.6 & $\mathrm{~m}$ \\
$\alpha_{y_{i}}$ & -1.6 & - \\
$\mathcal{C}$ & $1.0-6.0$ & $\mathrm{~m}^{-1}$ \\
Average Energy & 38.6 & $\mathrm{MeV}$ \\
\hline
\end{tabular}

parameters were sufficient to model the bunch compressor accurately without needlessly increasing the computational demand and time or suppressing small-scale features of the bunch. IMPACT-Z contains several dipole models, one that tracks only with space charge, and another that include an implementation of the 1-D model of steady-state CSR discussed above. 

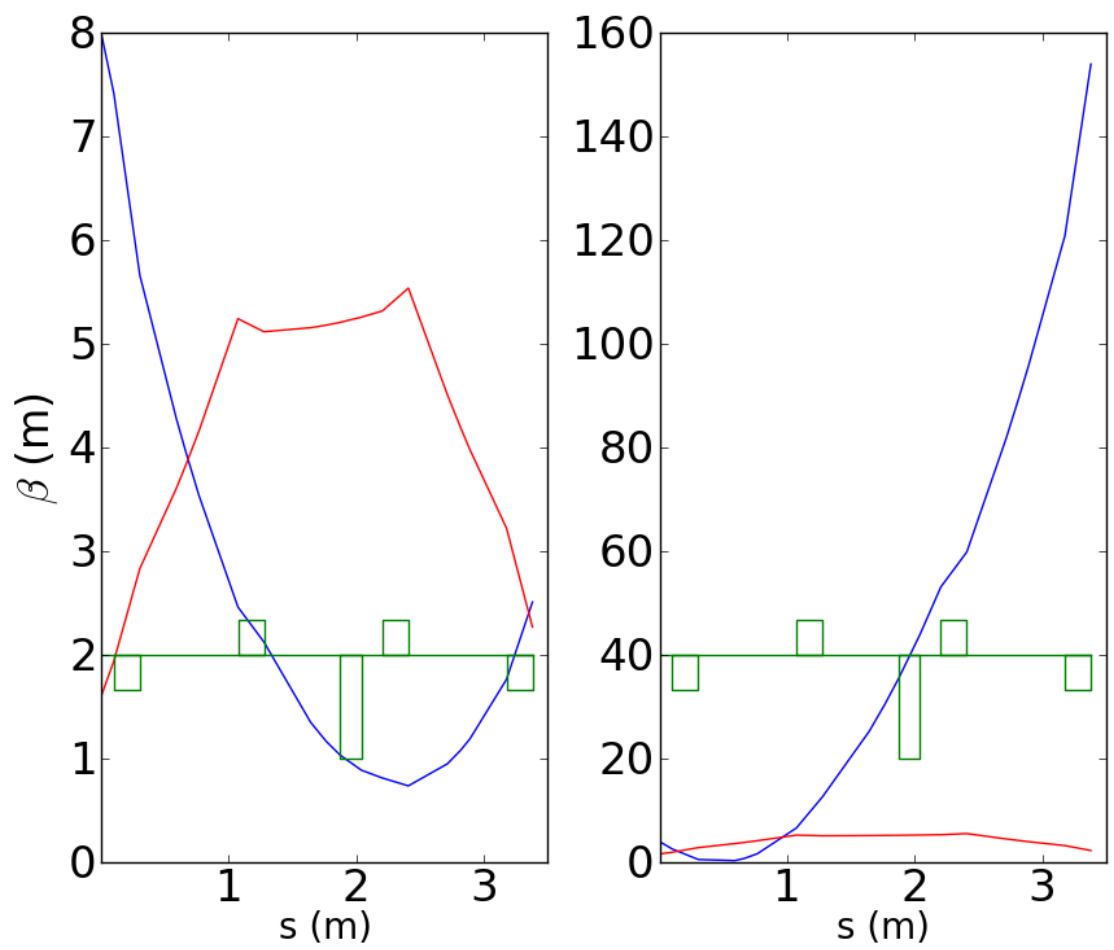

FIG. 7: Horizontal (blue) and verical (red) betatron functions evolution along BC1 obtained from ELEGANT. The left and right plots correspond respectively to points (A) and (B) in Fig. 6. The ordinate $s$ is the path-length throughout the chicane and $s=0$ corresponds to $0.1 \mathrm{~m}$ upstream of the entrance face of $\mathrm{B} 1$.

The first of the simulation parameters that we will discuss here is the number of bins to be used in the calculation of space charge effects, using a PIC algorithm. Having too few longitudinal bins, $N_{z}$, will smooth out the current distribution, reducing the effective peak current that drives emittance and energy spread growth, while having too many bins can introduce numerical noise that may lead to spurious effects. In addition a large number of bins increases the computational time of the simulations.

For these tests, we adjusted the $N_{z}$ by powers of 2 , as required by IMPACT-Z which uses a FFT algorithm to convolve the charge distribution with the free-space Green's function. The bunch used for the testing is a generated three-dimensional Gaussian distribution with rms parameters matched to the parameter computed for the "realistic" distribution generated by ASTRA. The number of particles in each of the simulations was increased proportional to the number of bins such that there were, on average, 5 macroparticles per three-dimensional cell. 
TABLE II: Number of macroparticles $N$ used as a function of the number of longitudinal bins $N_{z}$.

\begin{tabular}{cc}
\hline$N_{z}$ & $N$ \\
\hline \hline 64 & 81920 \\
128 & 163840 \\
256 & 327680 \\
512 & 655360 \\
1024 & 1310720 \\
2048 & 2621440 \\
\hline
\end{tabular}

With 16 cells in $x, 16$ cells in $y$, and 5 particles per cell, the total number of macroparticles $N$ for each of the simulations is shown in Table II. The value of $N_{z}$ was varied from 16 to 2048 and the corresponding simulations of BC1 included both the SC and CSR effects.

The number of particles to have an average of 5 particles per bin is the same order of magnitude as the number of particles that we have typically used in our simulations using realistic bunches (200k macroparticles). Given the lack of convergence in the emittance, there appears to be little benefit to increasing the $N_{z}$ above 256 bins. The bunch used for these tests is a generated Gaussian that has been matched to specified Courant-Snyder parameters and emittances, as the 200k-macroparticle distributions we export from AsTRA are insufficient for the type of parameter-optimizing performed here. The final LPS and current profiles are shown in Figs. 8 and 9, respectively, and the variance of the current profiles for various $N_{z}$ compared to that of $N_{z}=64$ are shown in Fig. 10. The final horizontal normalized emittance $\varepsilon_{n x}$ for these simulations are shown in 11.

The validity of these results is expected to depend on the precise distribution that is used. Because the beam is binned with spatial bins along the length of the bunch, distributions with long, sparsely populated tails may have different bin-number requirements to properly resolve their peak currents than more uniform and even distributions.

The other parameter that influences the simulations is the number of SC calculations done per beamline element. The sensitivity of the results on the number of SC kicks per element was found to be below $1 \%$ as long as we used four or more kicks per element. For this reason, our standard of four kicks per element is reasonable, and is used as the default 


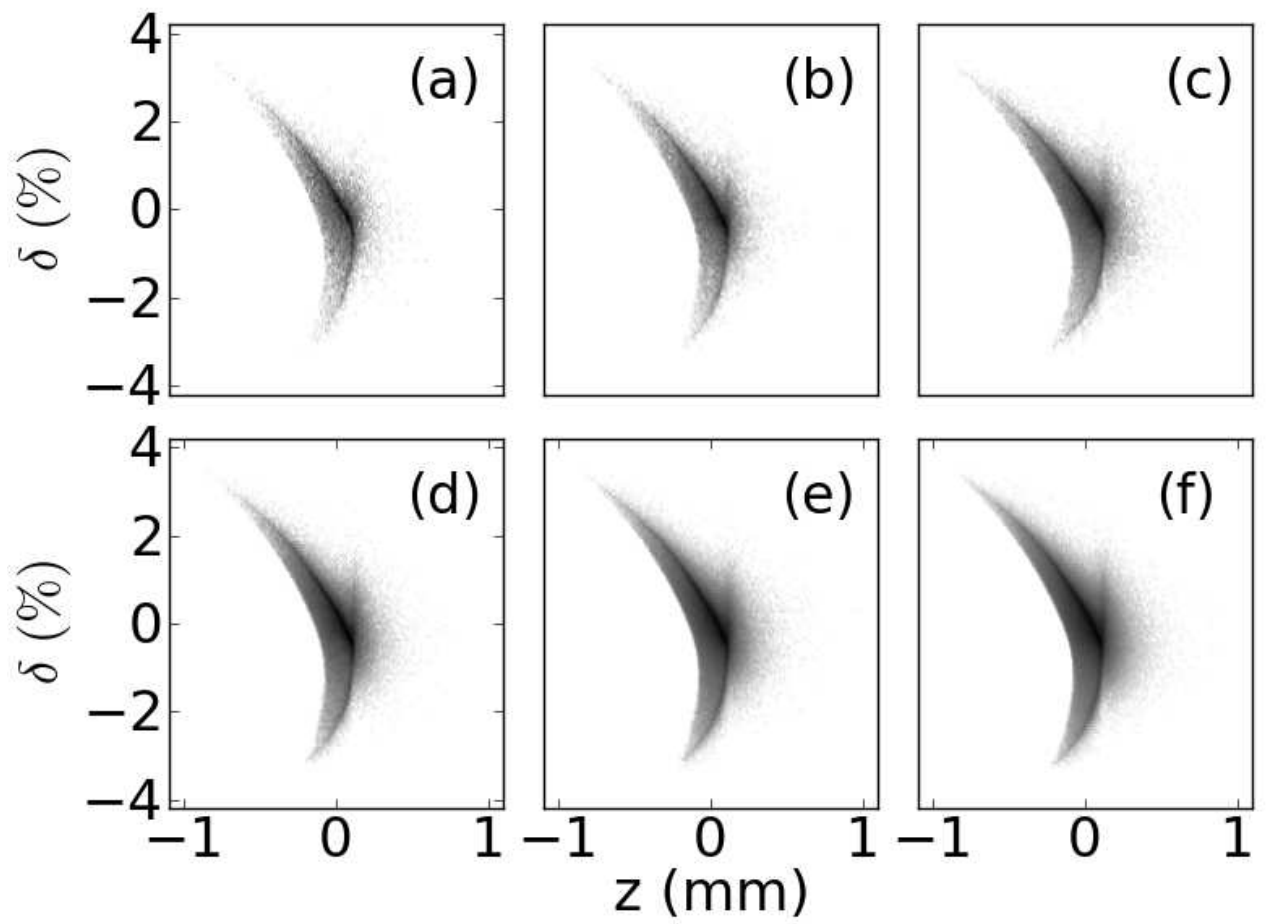

FIG. 8: LPS density plots at BC1 exit in IMPACT-Z varying the number of longitudinal spacecharge bins $N_{z}$, while keeping the number of particles per bin constant to $N /\left(N_{x} N_{y} N z\right)=5$, with $N_{z}$ set to 64 (a), 128 (b), 256 (c), 512 (d), 1024 (e) and 2048 (f) bins. Adjusting the $N_{z}$ has little impact on the longitudinal dynamics for these simulations.

value for the studies presented throughout this report.

\section{CSRTRACK PARAMETRIC STUDIES}

Using either CSRTRACK's P2P solver or its 1-D Projected model, the relevant simulation parameters (beyond basic descriptions of the dipoles and layout of the chicane) are the number and the size of the Gaussian sub-bunches, $\sigma_{B}$, used to represent the three-dimensional distribution. Due to the drastic increase in run time when using the P2P model, e.g, compared to IMPACT-Z, the studies presented here needed to be significantly more focused in scope.

The sub-bunch size determines the susceptibility to numerical noise. Going for this goal of distribution-smoothing likely dampens out any microbunching instabilities that could manifest in more complete simulations. However, accurately resolving microbunching in- 


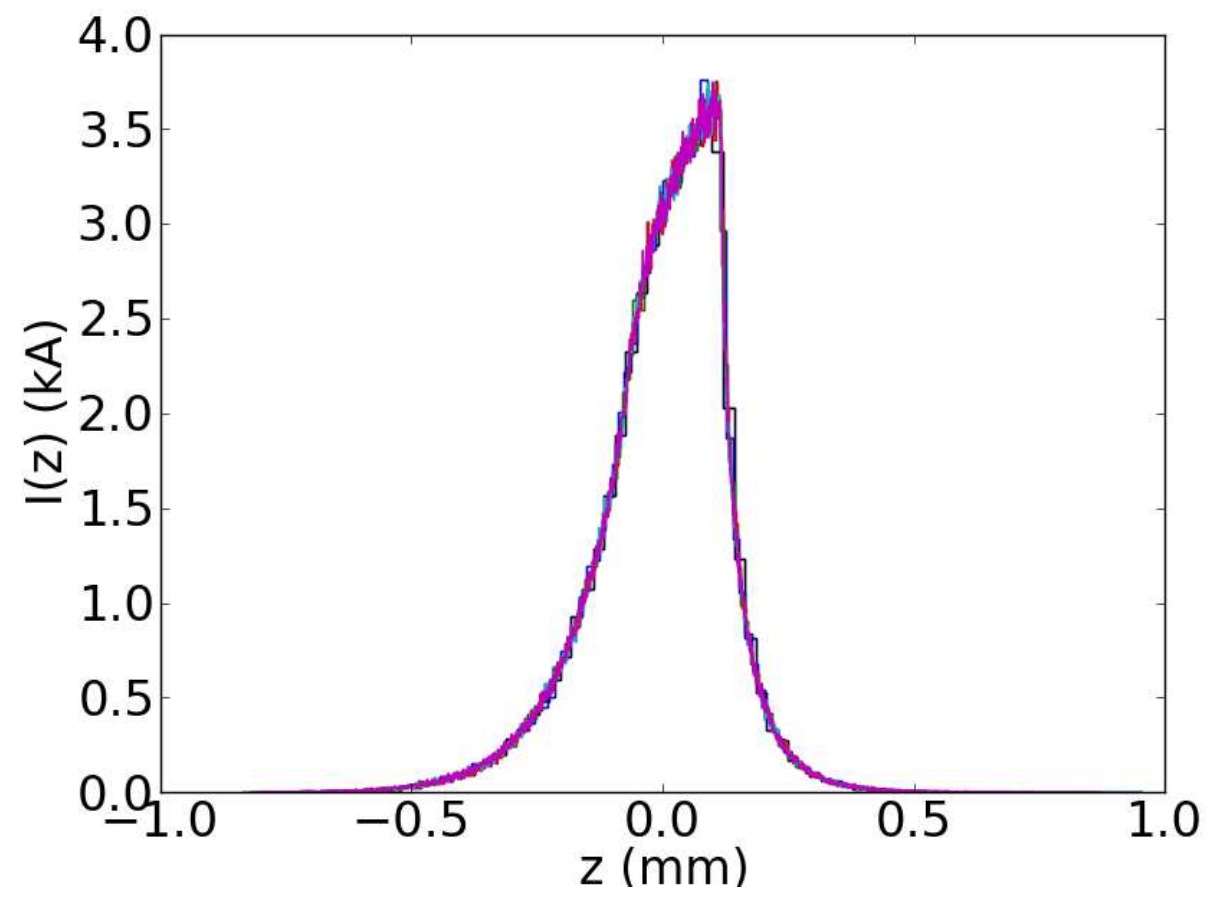

FIG. 9: Current profiles at BC1 exit in IMPACT-Z varying the number of longitudinal space-charge bins, while keeping the number of particles per bin constant by increasing the number of particles in the distribution, with $N_{z}$ set to 64 (red), 128 (blue), 256 (green), 512 (magenta), 1024 (yellow) and 2048 (black).

stabilities would be far more time and computationally intensive than those used for the studies presented in this Memo.

We used Fourier analysis as quick estimates of what gaussian sizes were appropriate. This entailed summing every gaussian sub-bunch over each projected axis to recreate the effective, smoothed distribution, using a method similar to that performed internally by CSRTRACK, as described in Eqs. 20 and 21. We then performed Fourier analysis to inspect for highfrequency noise, which would manifest itself in simulations as spurious forces. In cases where $\sigma_{B}$ is much smaller than the average inter-particle spacing, the fourier transformation would reveal noise. At the other extreme, where $\sigma_{B}$ is on the order of the bunch length $\sigma_{z}$, the effective length of the bunch is increased, which erroneously reduces space-charge and CSR effects. This led to the general procedure of trying to determine the shortest possible gaussian that does not suffer from spurious noise in the Fourier analysis.

We were also concerned that the focusing and compression would change the requirement 


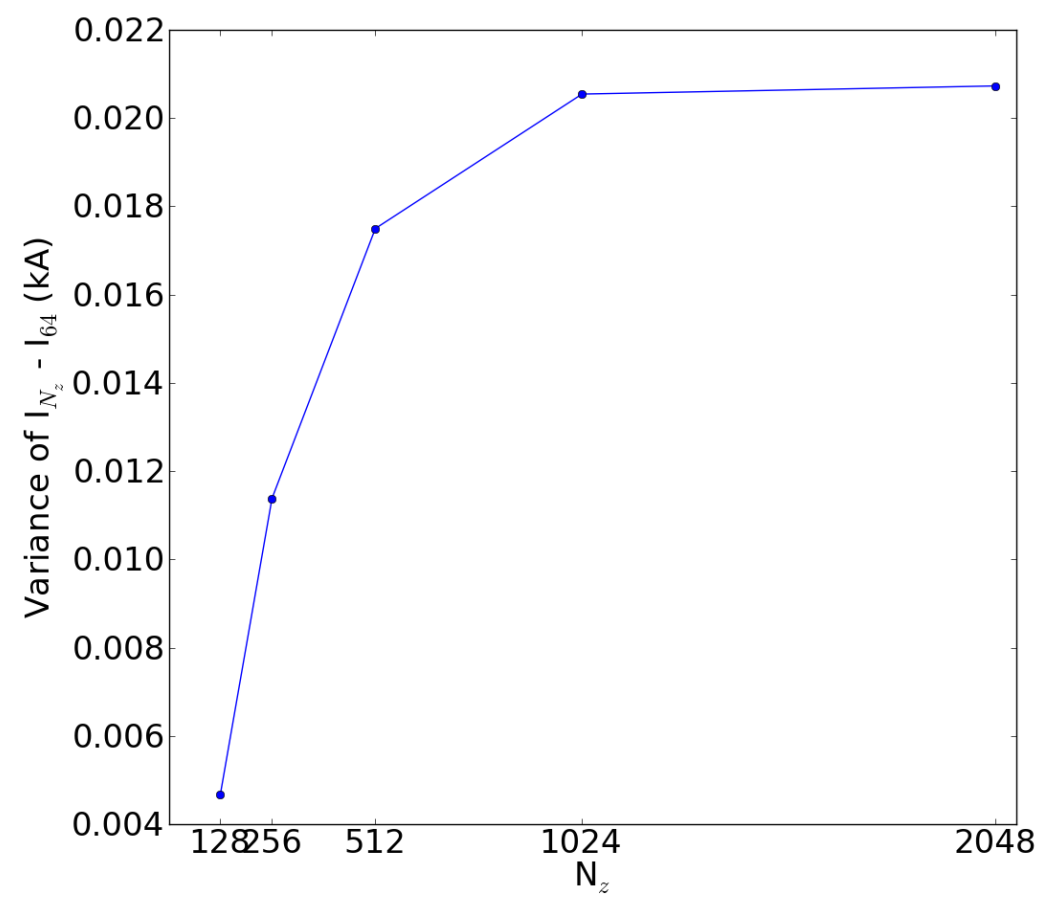

FIG. 10: Variance of $\mathrm{I}_{N_{z}}-\mathrm{I}_{64}$ for the data shown in Fig.9, where $\mathrm{N}_{z}$ is the number of longitudinal bins. Compared to the scale of the peak current, this variance is well under $1 \%$.

as the bunch passes through the compressor. Fortunately, CSRTRACK allows for setting the gaussian size as a variable of the bunch size at each time step. This is only applicable to the longitudinal and vertical dimension, and not the dimension of the bend. For the horizontal dimension, we chose it based on the final horizontal RMS size, as both CSR and SC effects are greater when the bunch lengths are shorter. The number of sub-bunches was then chosen to insure the transverse beam density does not display significant numerical noise.

With CSRTRACK's 1-D Projected model, we used 200k particles, so the required gaussian size needed to eliminate spurious noise was much smaller than the 3D simulations, which used $5 \mathrm{k}$ to $30 \mathrm{k}$ particles. In Table III, we present the final emittances and energy spreads for simulations using 200k particles, and variable Gaussian sub-bunches as some fraction of the total RMS length.

A Gaussian sub-bunch length of approximately $10 \%$ of the total RMS bunch size in $z$ and $y$ and $0.01 \mathrm{~mm}$ in $x$ was found to be appropriate for 10k particles. This then raises the question of what is appropriate when increasing and/or decreasing the size based on the number of particles used. 


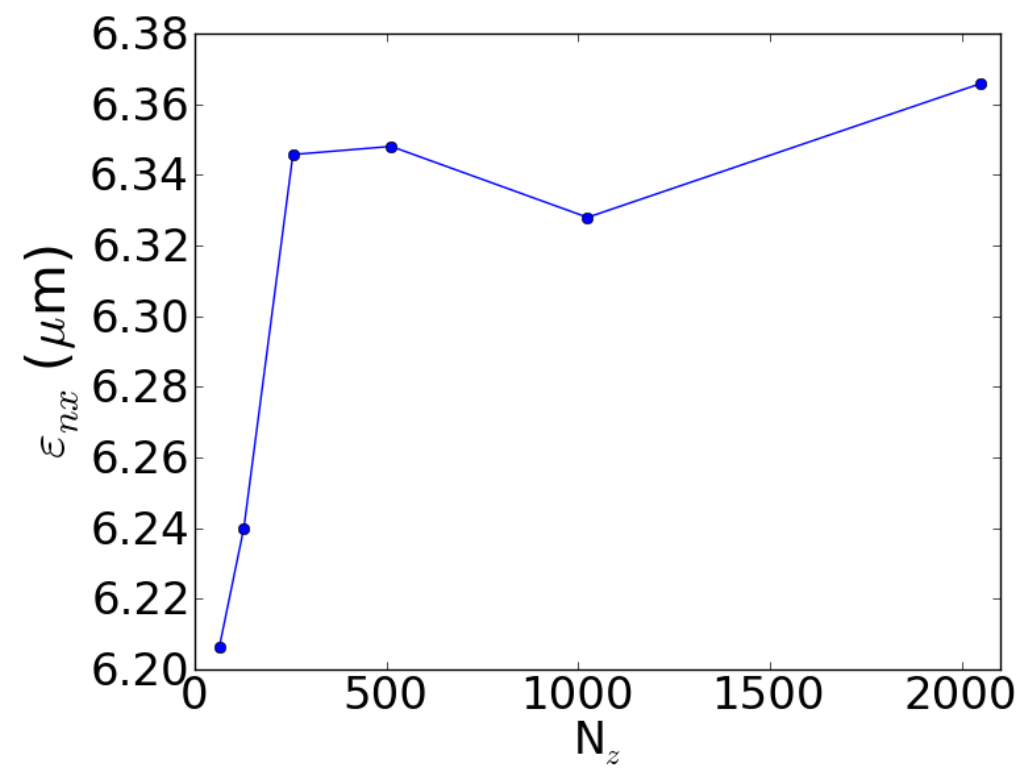

FIG. 11: $\varepsilon_{n x}$ BC1 exit in IMPACT-Z varying the number of longitudinal space-charge bins, $N_{z}$, while keeping the number of particles per bin constant, with $N_{z}$ set to $64,128,256,512,1024$ and 2048 number of bins.

When the sub-bunch size is around $0.1 \%$ of the RMS length or less, CSRTRACK's calculation fails to converge and the forces are not solved correctly, leading to erratic and/or outright broken behavior in which the CSR effects are not calculated correctly. This presents itself in outgoing emittances that are nearly what one would achieve with a single-particle (no CSR or LSC) simulation, as shown in Fig. 12. This corresponds to when the gaussian size is comparable to or smaller than the average inter-particle spacing, or

$$
<r>\approx 1 / n^{1 / 3}
$$

where $n \equiv N /\left(\sigma_{x} \sigma_{y} \sigma_{z}\right)$ is the macroparticle density. For a 3.2-nC bunch with the expected range of betatron functions and emittances, this is on the order of $0.1 \mathrm{~mm}$. When $\sigma_{B}$ is on the order of $\sigma_{z}$, the effective bunch length is increased and the emittance growth due to CSR is reduced. Values for $\sigma_{B}$ in the range of around 1\%-10\% of the RMS bunch length suffer from neither issue, and thus $5 \%$ of $\sigma_{z}$ is chosen to be the baseline for our 1DP model simulations.

For 3D simulations, such a comprehensive scan was too demanding, so we performed scattered simulations, with several different numbers and sizes for the Gaussian sub-bunches. 
TABLE III: Scan of Variable Size

\begin{tabular}{llll}
\hline$\%$ RMS length & $\varepsilon_{n x}$ & $\delta(\%)$ & $I_{\text {peak }}(\mathrm{kA})$ \\
\hline \hline $100 \%$ & 22.5 & 1.49 & 6.81 \\
$50 \%$ & 36.8 & 1.55 & 6.95 \\
$30 \%$ & 44.6 & 1.67 & 7.85 \\
$20 \%$ & 50.7 & 1.78 & 8.20 \\
$15 \%$ & 53.3 & 1.81 & 8.4 \\
\hline $10 \%$ & 54.9 & 1.83 & 8.04 \\
$5 \%$ & 54.5 & 1.87 & 8.78 \\
$1 \%$ & 55.3 & 1.87 & 7.73 \\
$0.5 \%$ & 55.4 & 1.87 & 7.11 \\
\hline
\end{tabular}

Table IV shows the results for the simulation models considered in this report. The settings and parameters for a 3.2-nC bunch with linearized LPS are shown.

To inspect the numerical variation from the process of randomly cutting up to $97.5 \%$ of the particles from the initial 200k distribution, we repeated the simulations several times with different sets of random particles. This effect, over the limited studies performed here, appears to be minimal.

Further studies will be performed as we use these simulations as the backbone for further studies for the ASTA facility.

The P2P model in CSRTRACK, with 10\% RMS gaussian sub-bunches, has little dependence on the number of macroparticles used over the range presented here, owing to the smoothing that the gaussian sub-bunches offer; see Fig. 13. Simulations with 5k particles take around an hour to perform, simulations with 10k particles take several hours (feasible for parametric scans), while the 30k-particle simulations take several days on a 48 -CPU cluster, which is reasonable for limited studies.

We choose $10 \mathrm{k}$ macroparticles with $\sigma_{B}=0.1 \sigma_{z}$ as the standard for our 3D simulations based on these results. Using a 48-CPU cluster, these simulations take around three-to-four hours to complete. 


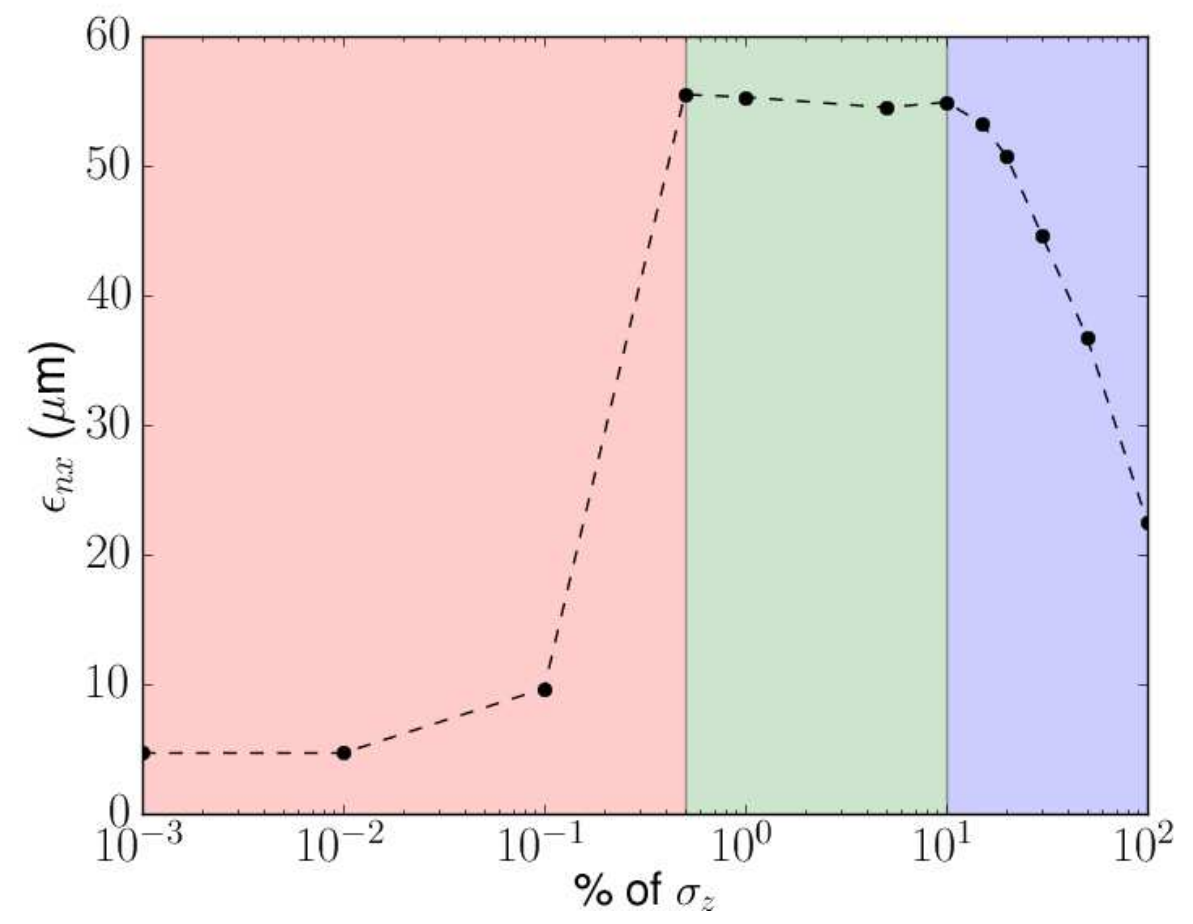

FIG. 12: $\varepsilon_{n x}$ BC1 exit in CSRTRACK with the 1DP model versus the size of the gaussian subbunches as a fraction of the RMS length, $\sigma_{z}$. The blue-shaded region corresponds to where $\sigma_{B} \sim \sigma_{z}$, which increases the effective bunch length and decreases the effects of the CSR. The green-shaded region corresponds to where $\sigma_{B}$ is sufficiently small to not increase the effective bunch length, which gives consistent results. The red-shaded region corresponds to where $\sigma_{B}<<\sigma_{z}$ and on the order of the average interparticle seperation, which causes CSRTRACK's solver to fail to converge resulting in an underestimation of the impact of CSR.

\section{CODE COMPARISONS AND PARAMETRIC STUDIES}

At ASTA, the bunch charge will be variable from a few pCs to several nCs. Some applications, e.g. the test of ILC subsystems for ILC bunch-parameters, calls for a $3.2 \mathrm{nC}$, while other experiments, such as high-brilliance X-ray generation via channeling radiation, require very low charge. It is therefore of interest to assess the performance of the BC1 over the anticipated range in charge. Following Ref. [18], we introduce the transverse brightness $B_{\perp}=\frac{\hat{I}}{4 \pi^{2} \varepsilon_{n x} \varepsilon_{n y}}$. The charges used and their initial transverse normalized emittances are shown in Table $\mathrm{V}$.

Each of the simulation codes discussed in this Memo use different methods of modeling the 
TABLE IV: Simulated beam parameters downstream of BC1 with IMPACT-Z ("IMPZ") and CSRTRACK ("CSRT") the model used is appended to the program's name. The parameter column and indicates the number of bins, or the absolute or relative (in \%) Gaussian particle size. These entries were generated for $\mathcal{C}=$ at $5.22 \mathrm{~m}^{-1}$, so that the bunch is near maximum compression.

\begin{tabular}{lccccc}
\hline Model & $N$ & Parameter & $\varepsilon_{x}(\mu \mathrm{m})$ & $\delta(\%)$ & $\hat{I}(\mathrm{kA})$ \\
\hline \hline IMPZ-SC+CSR $2 \cdot 10^{5}$ & $256^{a}$ & 77.5 & 3.00 & 7.99 \\
\hline CSRT-1DP & $2 \cdot 10^{5}$ & $1 \mu \mathrm{m}$ & 55.4 & 1.85 & 6.16 \\
CSRT-1DP & $2 \cdot 10^{5}$ & $10 \%$ & 54.9 & 1.83 & 8.04 \\
CSRT-1DP & $2 \cdot 10^{5}$ & $5 \%$ & 54.5 & 1.87 & 8.78 \\
CSRT-1DP & $2 \cdot 10^{5}$ & $1 \%$ & 55.3 & 1.87 & 7.73 \\
\hline CSRT-P2P & $5 \cdot 10^{3}$ & $10 \%$ & 101 & 2.81 & 6.37 \\
CSRT-P2P & $5 \cdot 10^{3}$ & $10 \%$ & 103 & 3.03 & 6.65 \\
CSRT-P2P & $1 \cdot 10^{4}$ & $10 \%$ & 102 & 2.89 & 6.57 \\
CSRT-P2P & $2 \cdot 10^{4}$ & $10 \%$ & 94.6 & 2.91 & 6.44 \\
CSRT-P2P & $3 \cdot 10^{4}$ & $10 \%$ & 98.4 & 2.86 & 6.44 \\
CSRT-P2P & $2 \cdot 10^{4}$ & $5 \%$ & 97.8 & 2.80 & 5.95 \\
\hline
\end{tabular}

${ }^{a}$ number of longitudinal bins; ${ }^{b}$ a different statistical sample of the $5 \times 10^{3}$ particles was used compared to previous line.

phase-space evolution of particles throughout the chicane, and account for collective effects in distinct ways. The most accurate simulations should be those that contain both space-charge effects and CSR effects. Both CSRTRACK's P2P model and IMPACT-Z's SC+CSR model contain both CSR and SC effects, and each of these simulations has an advantage over the other. IMPACT-Z allows for a greater number of macroparticles, while CSRTRACK models transverse CSR effects, as well as the fact that CSR fields follow the bunch downstream of a dipoles exit. In this section, we compare the behavior between the various models for various energy chirps, $\mathcal{C}$, and bunch charges, $Q$.

For each of the bunch charges specified earlier, we performed scans of the LPS chirp. For IMPACT-Z's SC and SC+CSR models, as well as CSRTRACK's 1-D Projected model, we use the range of $\mathcal{C} \in[1.0,6.0]$, and 200k macroparticles. For CSRTRACK P2P model, we focus 

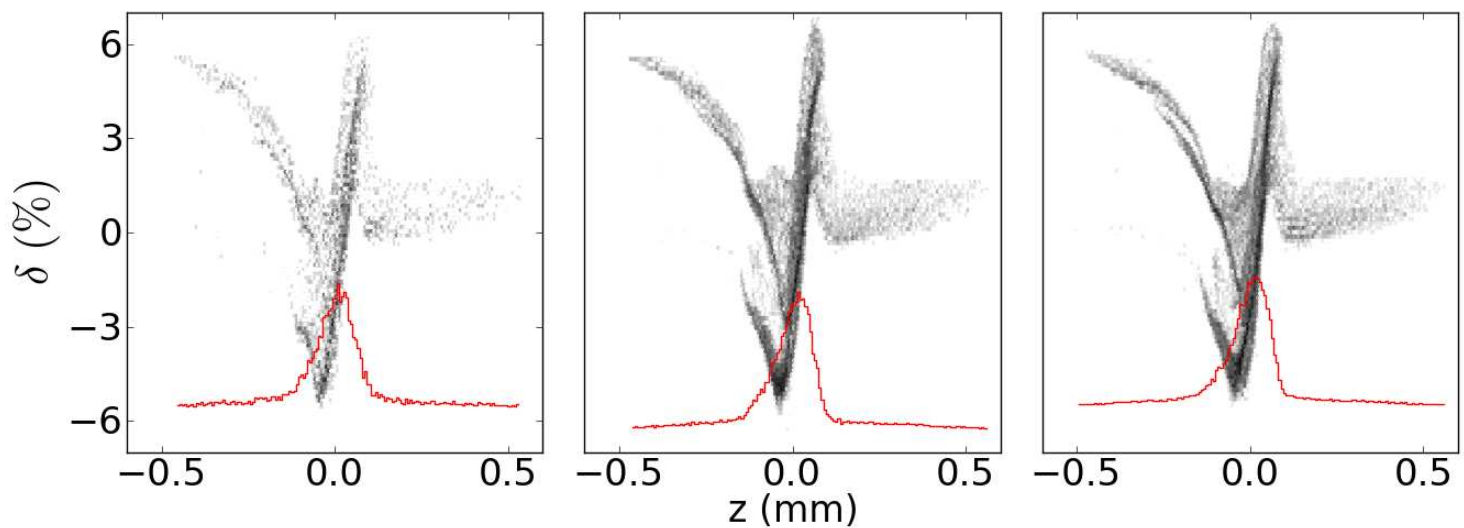

FIG. 13: LPS downstream of the bunch compressor and associated longitudinal charge distribution (red trace) in arbitrary units using CSRTRACK's P2P model, with 5k (left), 20k (middle), and 30k (right) macroparticles randomly downsampled from the same 200k particle distribution, with $\sigma_{B}=0.1 \sigma_{z}$, for initial LPS chirp of $5.22 \mathrm{~m}^{-1}$ using a $3.2 \mathrm{nC}$ bunch charge. The red line is the longitudinal current projection.

TABLE V: Initial transverse emittances for the charges used.

\begin{tabular}{cc}
\hline Bunch Charge $(\mathrm{nC})$ & $\varepsilon_{n x, y}(\mu \mathrm{m})$ \\
\hline 3.2 & 4.6 \\
1.1 & 2.25 \\
0.250 & 0.58 \\
0.020 & 0.294 \\
\hline
\end{tabular}

on a smaller range because of for the drastically increased execution-time required for these simulations, and use only $10 \mathrm{k}$ macroparticles with $\sigma_{B}=0.1 \sigma_{z}$.

Fig. 14 shows the bunch lengths downstream of BC1 versus LPS chirp, for each of the four bunch charges and for both of IMPACT-Z's models. Shorter bunch lengths can for lower bunch charges and without CSR.

In Fig. 15, we present the same Final transverse emittance $\varepsilon_{n x}(\mu \mathrm{m})$ versus LPS chirp using for four different bunch charges, using CSRTRACK's P2P model, CSRTRACK's 1DP model, ImPACT-Z's SC+CSR model, and ImPACT-Z's SC-only model. We also add an additional line that assumes that the emittance growths from SC (IZ) and CSR (1DP) are 

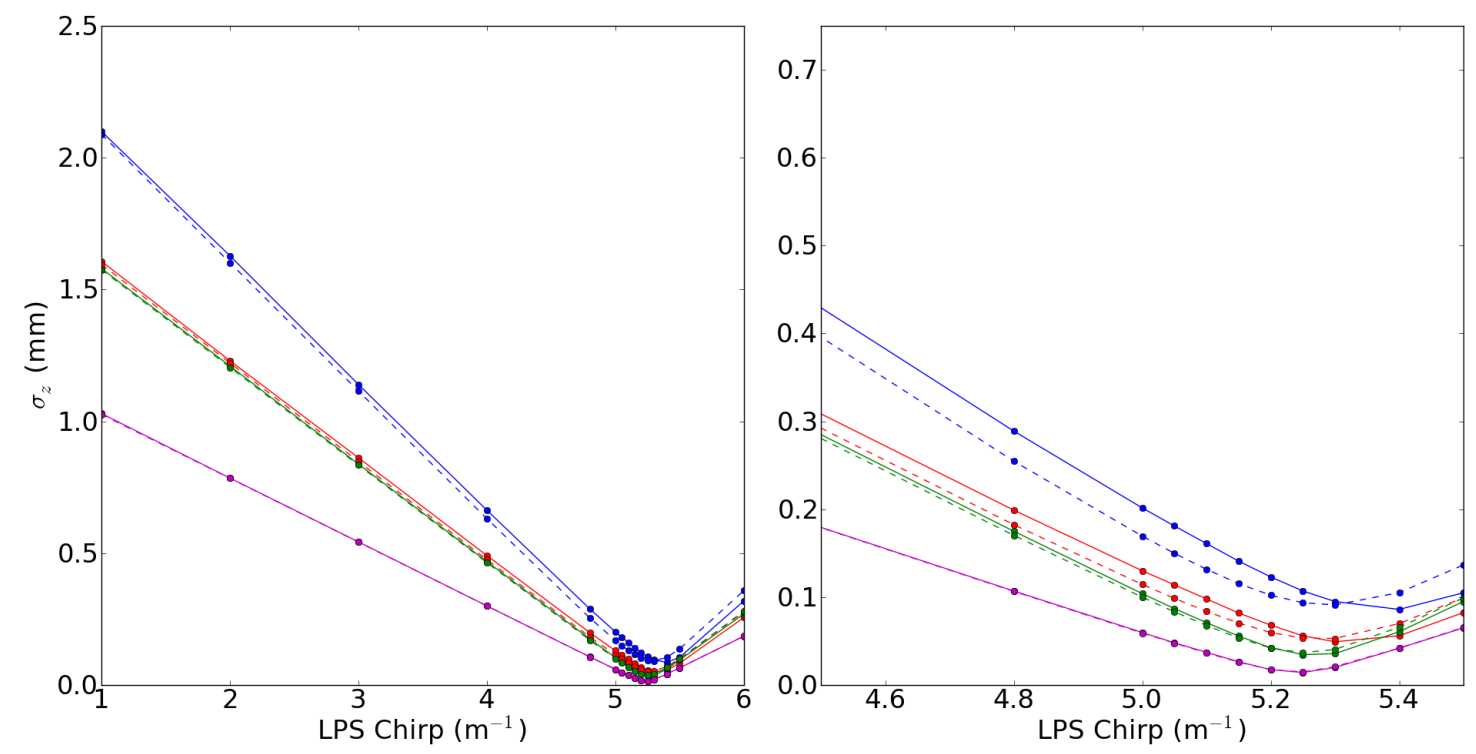

FIG. 14: Final bunch-length $\sigma_{z}(\mathrm{~mm})$ versus LPS chirp for various bunch charges using IMPACT-Z's $\mathrm{CSR}+\mathrm{SC}$ model (solid lines) and SC-only model (dashed lines), for 3.2-nC (blue), 1.0-nC (red), 250-pC (green), 20-pC (magenta) bunch charges, over the full range (left) and in the region near maximum compression (right).

added in quadrature with the initial transverse emittance (see Eq. 23-25) as a rudimentary estimate of what we should see from models that included both SC and CSR. There is overall good agreement between this simple model and IMPACT-Z's SC+CSR model, and the lower the charge the better the agreement. In Fig. 16, we show $\varepsilon_{n y}$ rather than $\varepsilon_{n x}$, which is not increased at all in the CSRTRACK simulations due to their neglection of the vertical dimension.

$$
\begin{aligned}
\Delta \varepsilon_{S C} & =\sqrt{\Delta^{2} \varepsilon_{f(S C)}-\varepsilon_{n i}^{2}} \\
\Delta \varepsilon_{C S R} & =\sqrt{\Delta^{2} \varepsilon_{f(C S R 1 D)}-\varepsilon_{n i}^{2}} \\
\varepsilon_{n f} & =\sqrt{\varepsilon_{n i}^{2}+\Delta^{2} \varepsilon_{S C}+\Delta^{2} \varepsilon_{C S R}}
\end{aligned}
$$

At higher bunch charges, CSR has a greater contribution to emittance-dilution, while at low bunch charges, the space-charge effects are a greater contribution, as demonstrated in the reversal of the lines representing Impact-Z's SC-only model and CSRTRACK's 1DP model. 

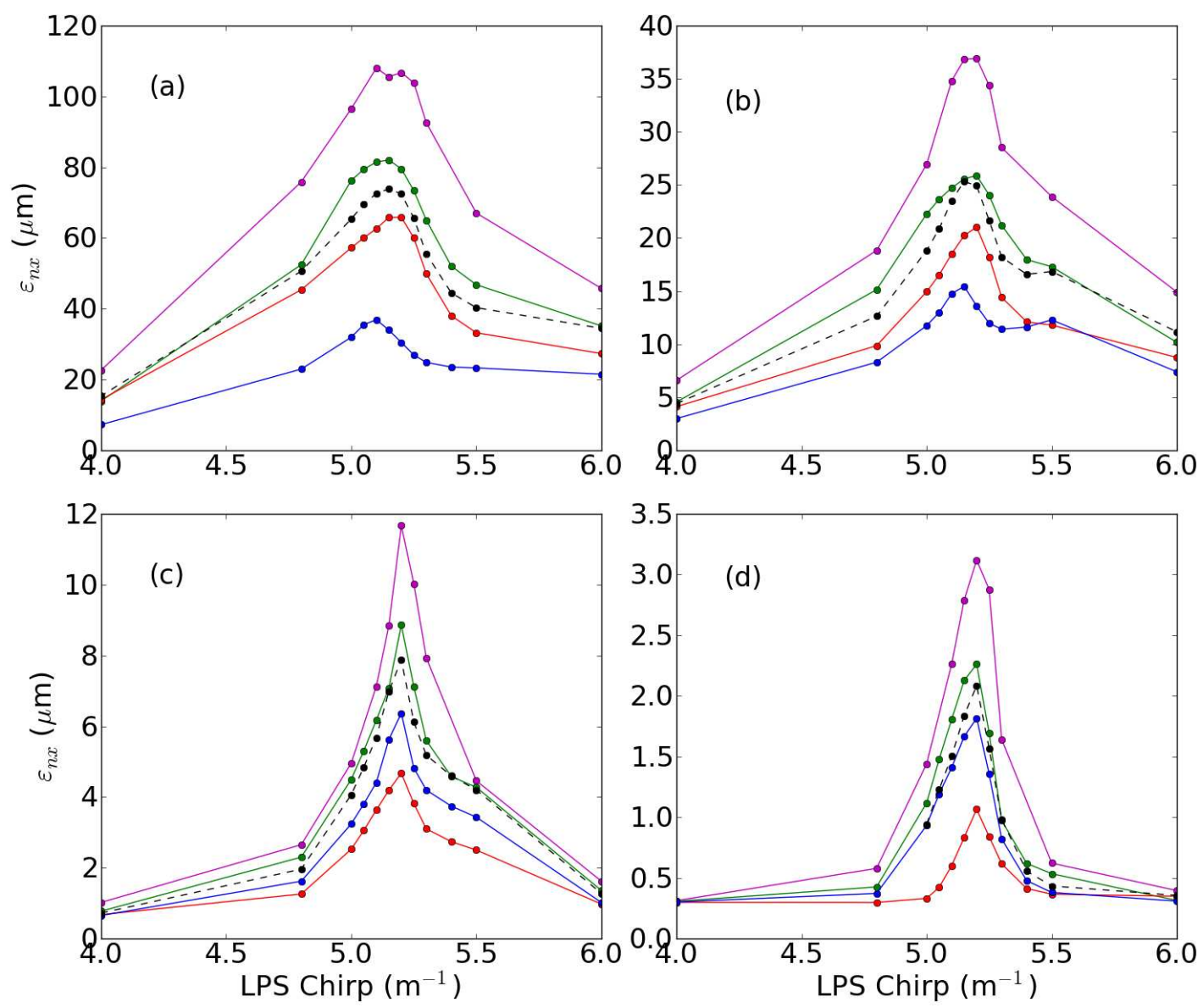

FIG. 15: Final $x$ emittances for each of the different bunch charges with Impact-Z's SC-only model (blue), CSRTRACK's 1D CSR-model (Red), and Impact-Z's SC+CSR model (Green), and CSRTraCK's P2P model (magenta), for 3.2-nC (a), 1-nC (b), 250-pC (c), and 20-pC (d) bunch charges. The dashed black line is the quadratic sum $\sqrt{\varepsilon_{n x i}^{2}+\Delta_{\varepsilon_{S C}}^{2}+\Delta_{\varepsilon_{C S R}}^{2}}$ as described in Eq. 23.

Comparisons of the LPS for all four bunch charges in both IMPACT-Z's SC+CSR model and CSRTRACK's P2P model, at an energy chirp of $5.2 \mathrm{~m}^{-1}$ are shown in Figs. 17, showing that the final LPS from each of the simulation codes shows the same fine-structure behavior, albeit at a smaller scale for the lower-charge simulations.

The agreement in emittance growth between the SC+CSR and P2P models is reasonable, and a visual comparison of the longitudinal phase-spaces shows many of the similar characteristics, shown in Fig. 18. The CSRTRACK simulations are presumably the more accurate 

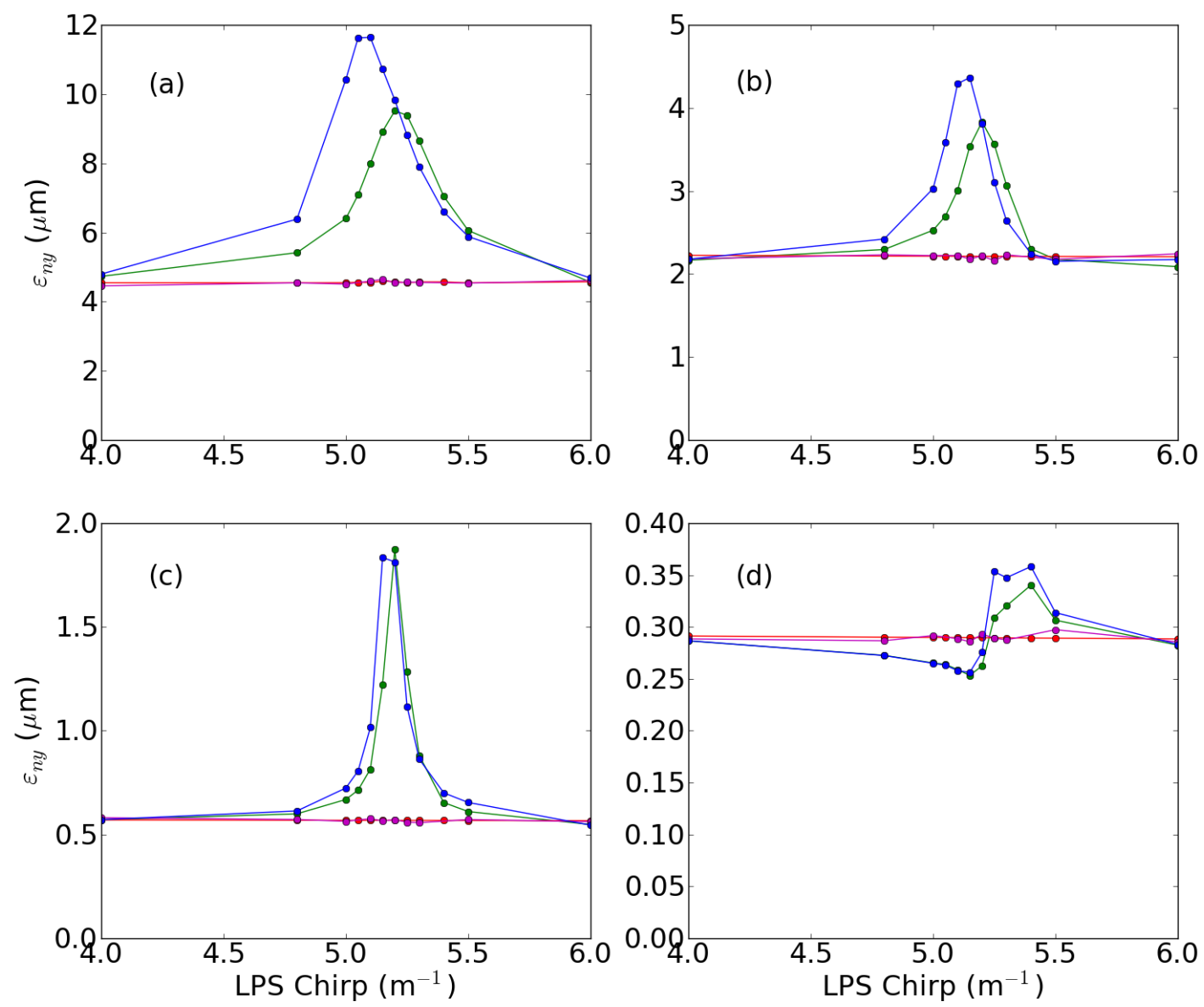

FIG. 16: Final $y$ emittances for each of the different bunch charges with Impact-Z's SC-only model (blue), CSRTRACK's 1D CSR-model (Red), and Impact-Z's SC+CSR model (Green), and CSRTraCK's P2P model (magenta), for 3.2-nC (a), 1-nC (b), 250-pC (c), and 20-pC (d) bunch charges. Neither model in CSRTRACK models the vertical transverse forces.

of the two, but IMPACT-Z's simulations take only a small fraction of the time, with vastly more particles.

As mentioned earlier in this paper, we use the transverse brightness, $B_{\perp}=\frac{\hat{I}}{4 \pi^{2} \varepsilon_{n x} \varepsilon_{n y}}$, as a figure of merit for the beam. Fig. 19 shows the peak currents for each charge and simulation code as a function of the energy chirp. Fig. 20 then takes those peak currents and uses it and the emittances shown earlier to calculate the $B_{T}$. Lastly, Fig. 21 takes the maximum brightness for each simulation and charge, and confirms that high charges result in lower 

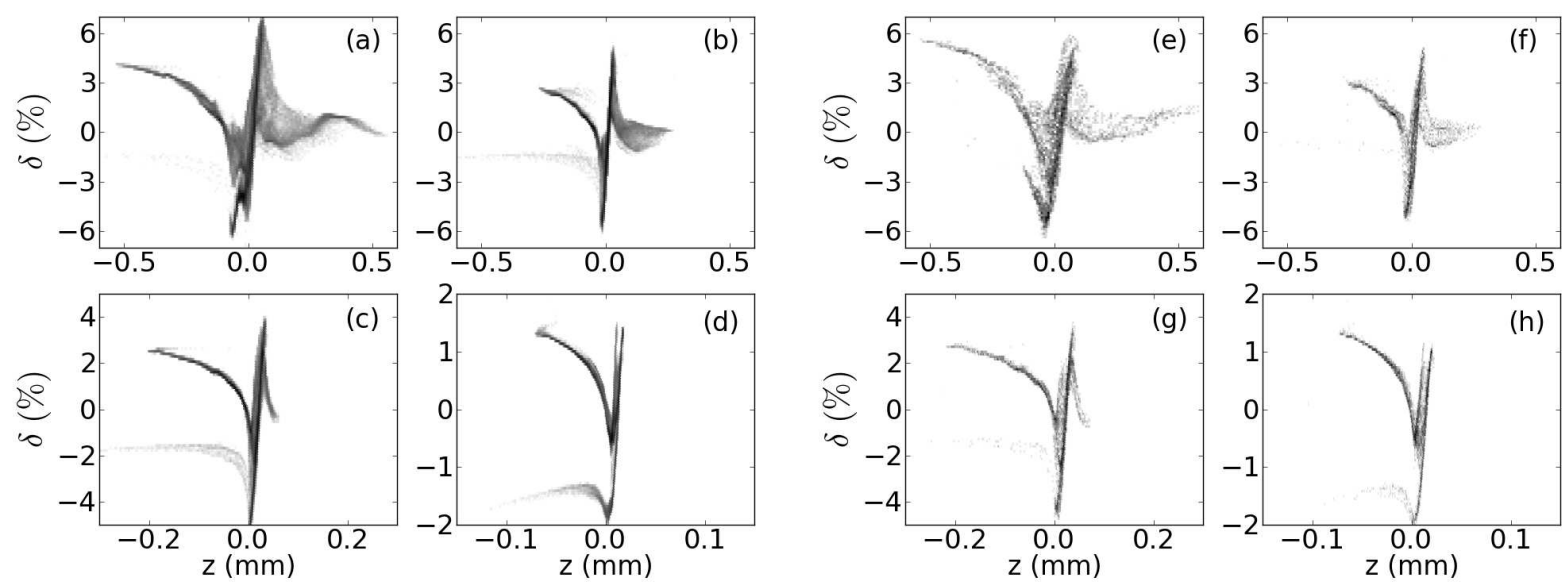

FIG. 17: LPS at BC1 exit for IMPACT-Z's (left set) and CSRTRACK's (right set) 3D models, for 3.2-nC (a,e), 1-nC (b,f), 250-pC (c,g), and 20-pC (d,h) bunch charges, zoomed in to show details. Note that the horizontal and vertical axis ranges are different for each plot.

values of $\hat{B_{\perp}}$.

Greater brightnesses can be achieved using smaller charges than with greater charges, as the greater peak currents that come with higher bunch charges are not great enough to compensate for the increase emittance growth for this injector. Likewise, the highest brightnesses may not come from maximum compression, again due to the peak currents there driving the greatest emittance growth. For user-experiments at ASTA, bunch charges lower than $3.2 \mathrm{nC}$ are suggested if high brightness is required.

\section{A. High-Energy Limit}

To help demonstrate the differences between the SC and CSR models implemented in the codes, we increase the energy of the particles to $400 \mathrm{MeV}$ while keeping the emittances the same, and perform the same scan of LPS chirp as described earlier with IMPACT-Z's SC+CSR and CSRTRACK's P2P model using the same chicane parameters.

As transverse SC effects are proportional to $\gamma^{-2}$, SC effects are almost completely suppressed at $400 \mathrm{MeV}$, with IMPACT-Z's SC model showing near-zero emittance growth. The other three simulations show varying degrees of emittance growth due to the different models used by each one- IMPACT-Z's version of the Saldin method, CSRTRACK's 1DP's kernel 

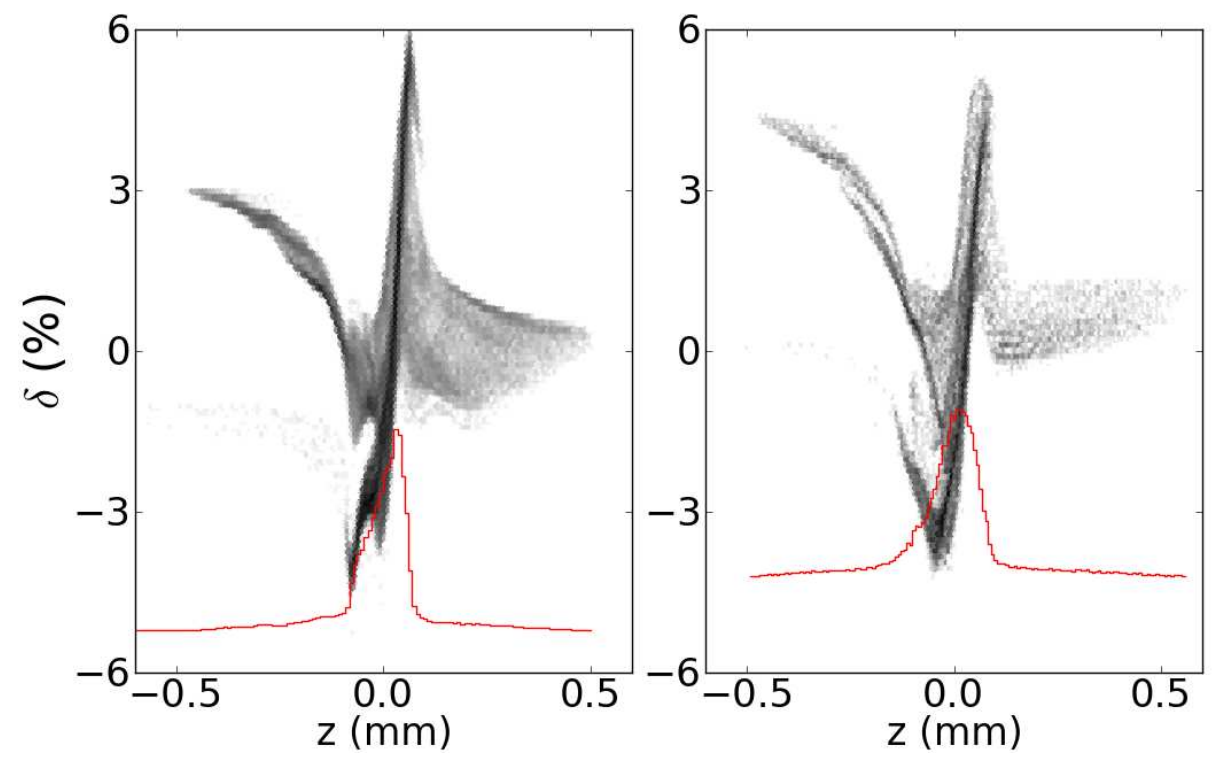

FIG. 18: LPS at the end of the bunch compressor and associated longitudinal charge distribution (red trace) in arbitrary units, with IMPACT-Z using 200k particles (left) and CSRTRACK's P2P model with 30k particles and 10\% RMS sub-gaussians (right), for an initial LPS chirp of $5.22 \mathrm{~m}^{-1}$ and bunch charge of $3.2 \mathrm{nC}$.

method, and CSRTRACK's multidimensional model. Due to the suppression of SC effects, we can infer that the resulting discrepancies are only functions of the different SC models. While CSRTRACK's 1-D Projected and Particle-to-Particle models are in good agreement, the Saldin model for CSR implemented in IMPACT-Z appears to be significantly weaker than the energy-dependent model used in CSRTRACK. This may be a result of both of CSRTRACK's models including some transverse forces, which are absent from the Saldin 1D model.

\section{FLAT BEAMS}

If the photocathode is immersed in a large axial magnetic field, the $x$ and $y$ distributions are correlated, and a set of skew quadrupoles downstream of the CAV2 may remove this correlation, resulting in a "flat beam" with a large emittance aspect ratio, $\frac{\varepsilon_{n x i}}{\varepsilon_{n y i}}[19-21]$. User-experiments at ASTA may benefit from using flat beams, and it may be possible to mitigate the emittance growth in the bunch compressor by having a beam that is wide in 

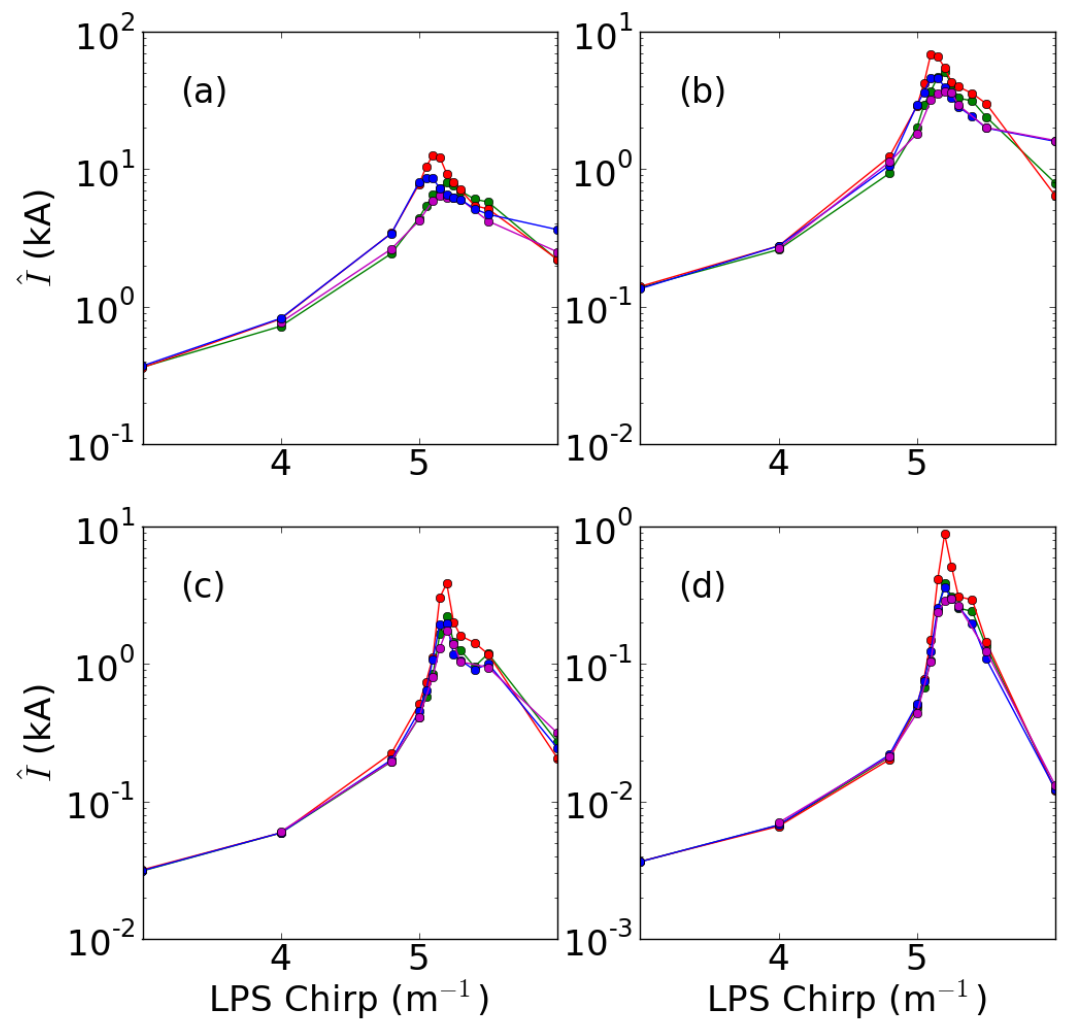

FIG. 19: Peak currents $\hat{I}$ versus energy chirp for ImPACT-Z's SC+CSR (green), ImPACT-Z's SC (blue), CSRTrack's 1DP (red), and CSRTrack's P2P (magenta) models, for $3.2 \mathrm{nC}$ (a), $1.0 \mathrm{nC}$ (b), $250 \mathrm{pC}(\mathrm{c})$, and $20 \mathrm{pC}(\mathrm{d})$ bunch charges

the direction of the chicane bend. In this section, we explore the behavior of flat beams in the low-energy bunch compressor at ASTA, using the emitance ratios shown in Table VI.

Due to the wider beam, it is anticipated that the projected CSR model is inadequate, thus we use CSRTRACK's P2P model to simulate the flat beams. The parameters used for flat beam simulations follow those used in the previous section, with one exception. In the previous section, we used an $x$ size $\sigma_{B x}$ as $0.1 \mathrm{~mm}$, or on the order of $10 \%$ of the average RMS size. Due to the much greater transverse dimension chosen here, we had to use a greater Gaussian size, so for these simulations $\sigma_{B x}=0.2 \mathrm{~mm}$. The results of these simulations are shown in Figs. 23-25

There are two advantages to using a flat beam that are supported by these results. Using a flat beam reduces the transverse emittance growth, both in absolute terms and as a relative 

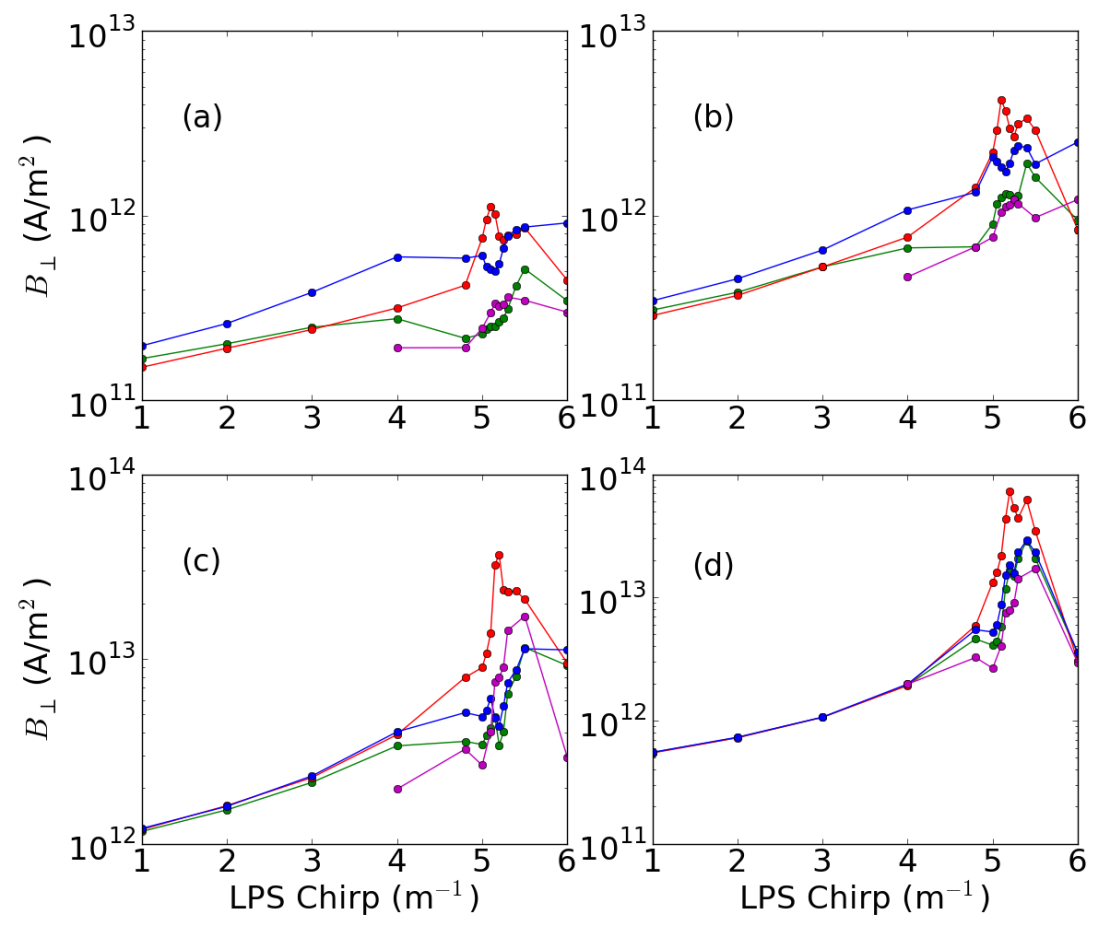

FIG. 20: Peak transverse brightness $B_{\perp}=\frac{\hat{I}}{4 \pi^{2} \varepsilon_{n x} \varepsilon_{n y}}$ versus energy chirp for IMPACT-Z's SC+CSR (green), ImPACT-Z's SC (blue), CSRTraCK's 1DP (red), and CSRTRACK's P2P (magenta) models, for $3.2 \mathrm{nC}(\mathrm{a}), 1.0 \mathrm{nC}(\mathrm{b}), 250 \mathrm{pC}(\mathrm{c})$, and $20 \mathrm{pC}(\mathrm{d})$ bunch charges

TABLE VI: Initial parameters for different aspect ratios.

\begin{tabular}{ccc}
\hline Aspect Ratio $\varepsilon_{n x i}(\mu \mathrm{m})$ & $\varepsilon_{n y i}(\mu \mathrm{m})$ \\
\hline \hline 1 & 5 & 5 \\
25 & 25 & 1 \\
100 & 50 & 0.5 \\
400 & 100 & 0.25 \\
\hline
\end{tabular}

fraction. Using a flat beam with a much larger $\varepsilon_{n x i}$ gives a final $\varepsilon_{n x f}$ on roughly the same order, regardless of the initial emittance, while allowing for much smaller $\varepsilon_{n y f}$. Furthermore, in user experiments where $\varepsilon_{n x}$ is not of much relevance, the growth that occurs in BC1 is of even less relevance. 


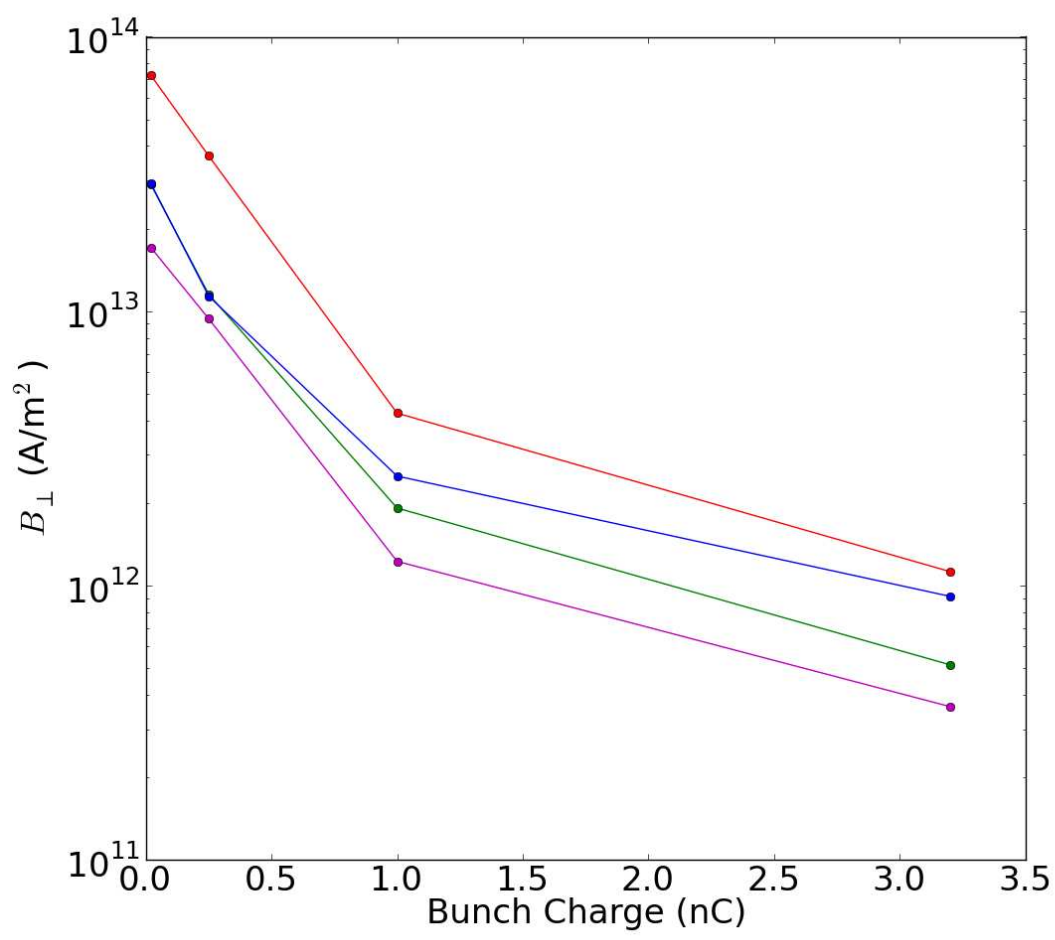

FIG. 21: Maximum peak transverse brightness $B_{\perp}=\frac{\hat{I}}{4 \pi^{2} \varepsilon_{n x} \varepsilon_{n y}}$ versus bunch charge for IMPACTZ's SC+CSR (green), ImPaCt-Z's SC (blue), CSRTraCK's 1DP (red), and CSRTraCk's P2P (magenta) models. Each data point is a maxima from each line in Fig. 20

\section{BUNCH SHAPING}

Over-compression may allow one to create current profiles that are well matched to some potential AARD applications. For instance, linearly-ramped current profiles can be used to explore beam-driven acceleration methods with an improved transformer ratio [22-24]. Likewise, a double-peaked distribution could prove useful to investigate and optimize the longitudinal beam dynamics along the ASTA accelerator. Such a double-peaked distribution could also support the test of beam driven acceleration where the first bunch generates wakefields with sub-mm wavelengths and a second, lower-charge "witness" bunch experiences the accelerating fields produced by the first bunch. Such a distribution turns out to be readily achievable with BC1, as shown in Fig. 26. The distribution displayed in Fig. 26 is obtained by over-compressing a $3.2-\mathrm{nC}$ bunch with $\mathcal{C}=5.50 \mathrm{~m}^{-1}$. The obtained bunch separation $\Delta z \simeq 0.3 \mathrm{~mm}$ is consistent with the period of wakefields produced in dense plasma [25] or 

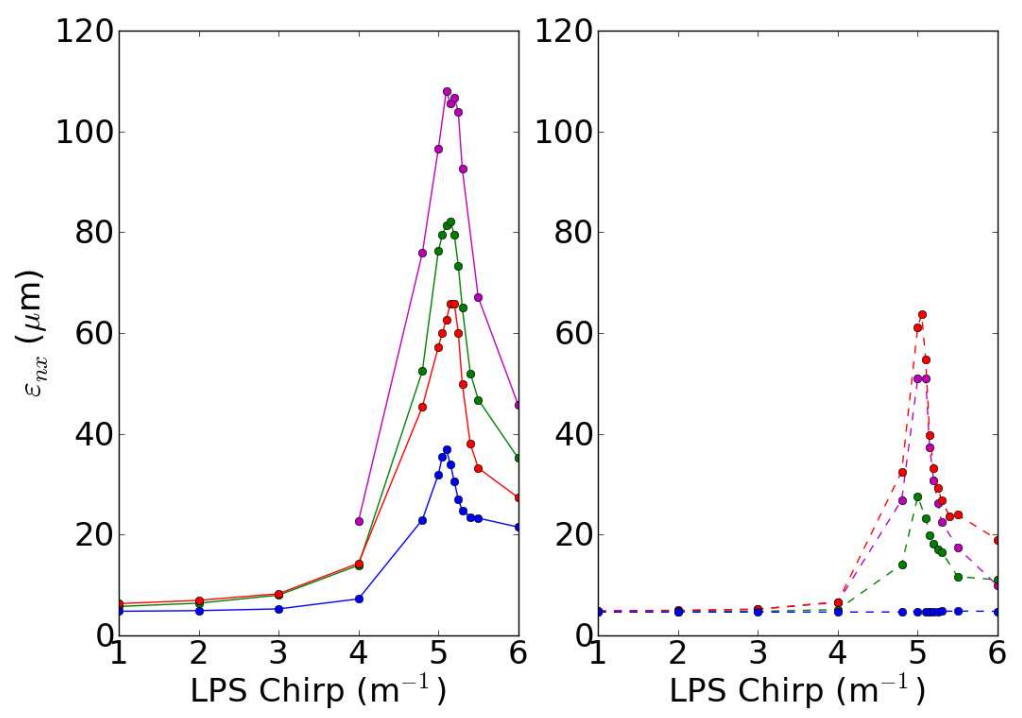

FIG. 22: Final transverse emittance $\varepsilon_{n x}(\mu \mathrm{m})$ versus LPS chirp using IMPACT-Z's SC+CSR (green), Impact-Z's SC (blue), CSRTrack's 1DP (red), and CSRTRACK's P2P (magenta) models, at 38.6 MeV (left) and $400 \mathrm{MeV}$ (right). SC-driven emittance growth is almost totally mitigated at such high energies, while the three models of CSR offer varying emittance growths, with reasonable agreement.

dielectric-wakefield waveguides [26].

\section{CONCLUSION}

A variety of simulation codes and models were tested for the design parameters of a lowenergy bunch compressor at the ASTA facility, over a wide-range of simulation parameters, including bin-size, number of particles, energy chirp, and length of gaussian sub-bunches, and across various models in IMPACT-Z and CSRTRACK. There is reasonable agreement between the two SC+CSR models, and their final LPSs show much of the same behavior. Their accuracy will be compared against the early experimental results when the ASTA facility begins operation next year.

The large emittance growth and subsequent loss of brightness at high-compression and high bunch charges indicates that user experiments that require high-brightness beams should explore using lower bunch charges and/or compression should be staged with a second bunch compressor at a higher-energy after subsequent acceleration in the cryomodules. 

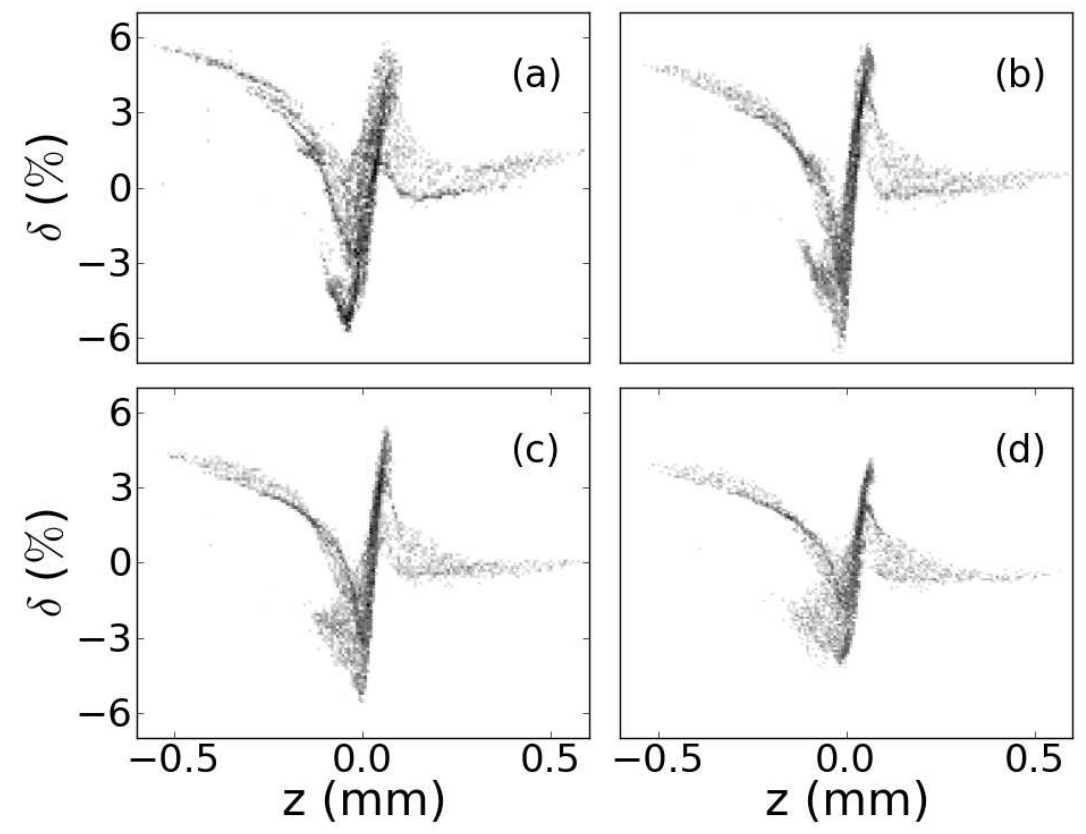

FIG. 23: Final LPS for initial emittance ratios of 1 (a), 25 (b), 100 (c), and 400 (d).

\section{ACKNOWLEDGEMENT}

This work was supported by LANL Laboratory Directed Research and Development (LDRD) program, project 20110067DR and by the U.S. Department of Energy under Contract No. DE-FG02-08ER41532 with Northern Illinois University and under Contract No. DE-AC02-07CH11359 the Fermi Research Alliance, LLC. 


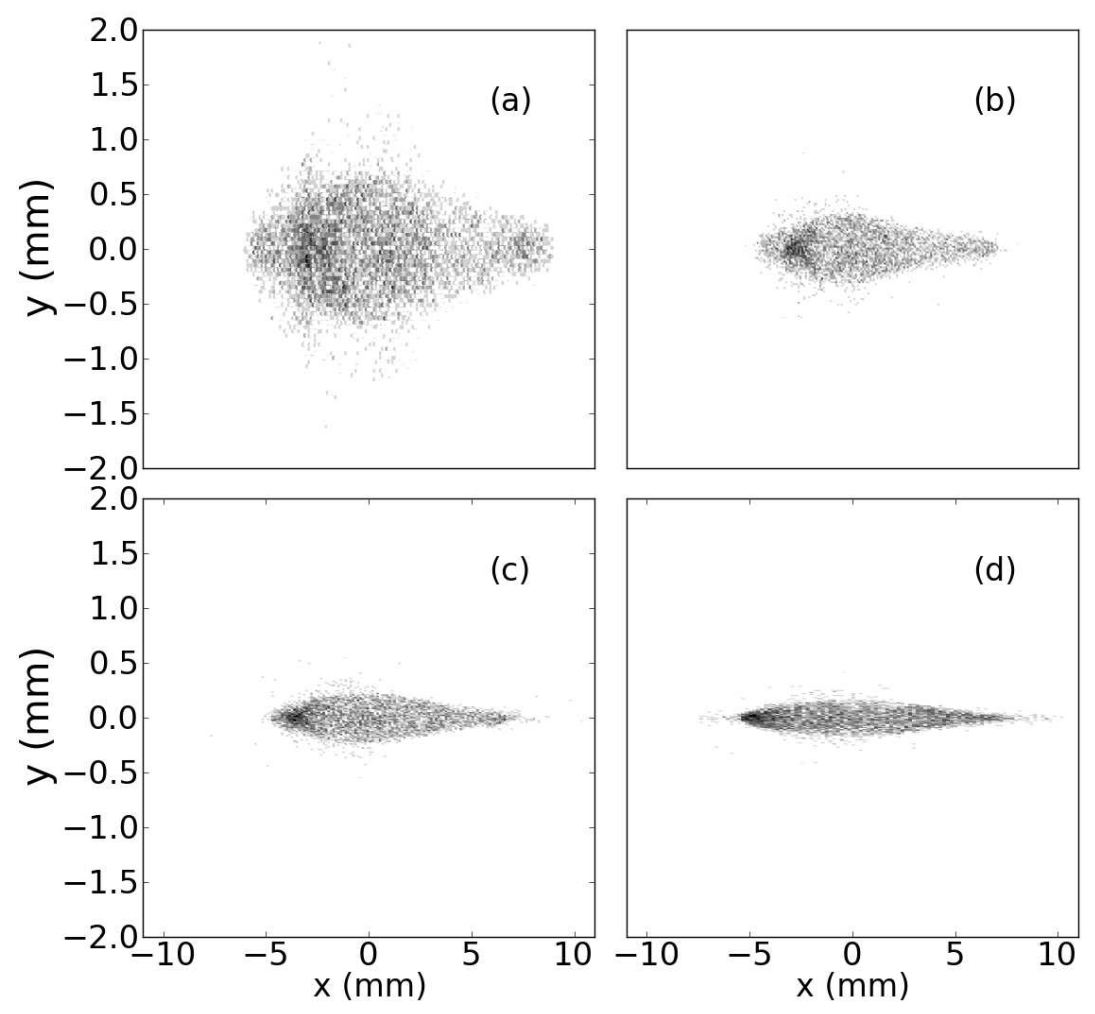

FIG. 24: Final $x-y$ profiles for initial emittance ratios of 1 (a), 25 (b), 100 (c), and 400 (d).
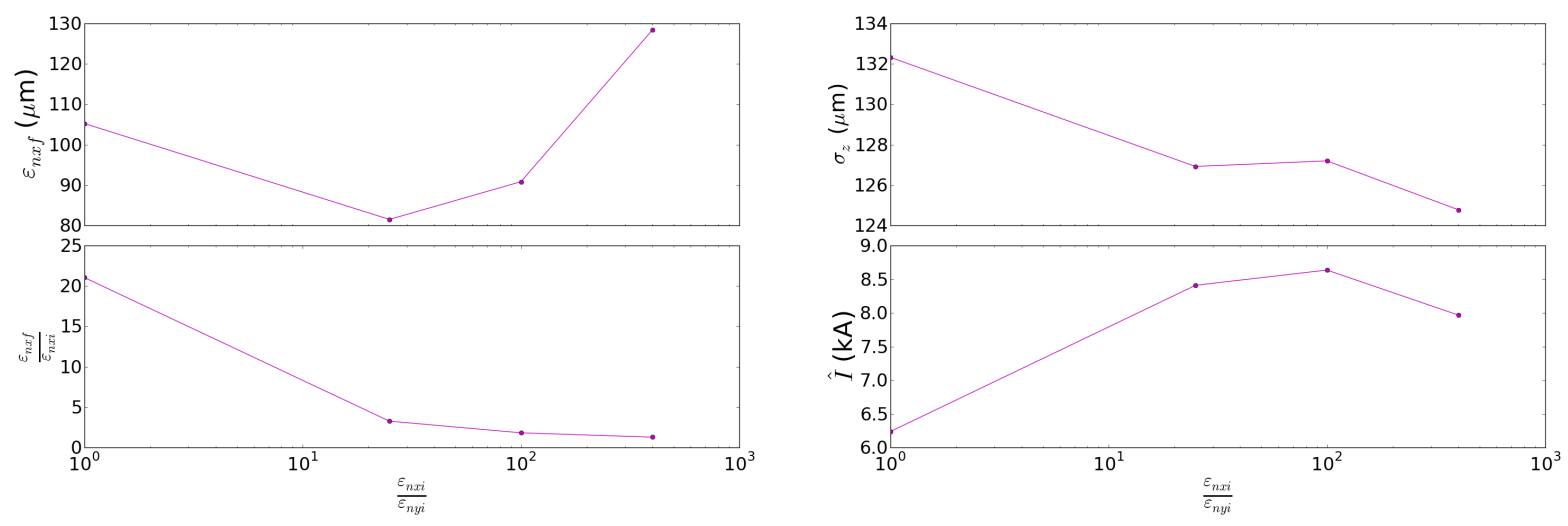

FIG. 25: Final transverse emittances $\varepsilon_{n x f}$, relative emittance growths $\frac{\varepsilon_{n x f}}{\varepsilon_{n x i}}$, bunch lengths $\sigma_{z}$, and peak currents at the exit of $\mathrm{BC} 1$ as functions of the initial aspect ratio, $\frac{\varepsilon_{n x i}}{\varepsilon_{n y i}}$ 

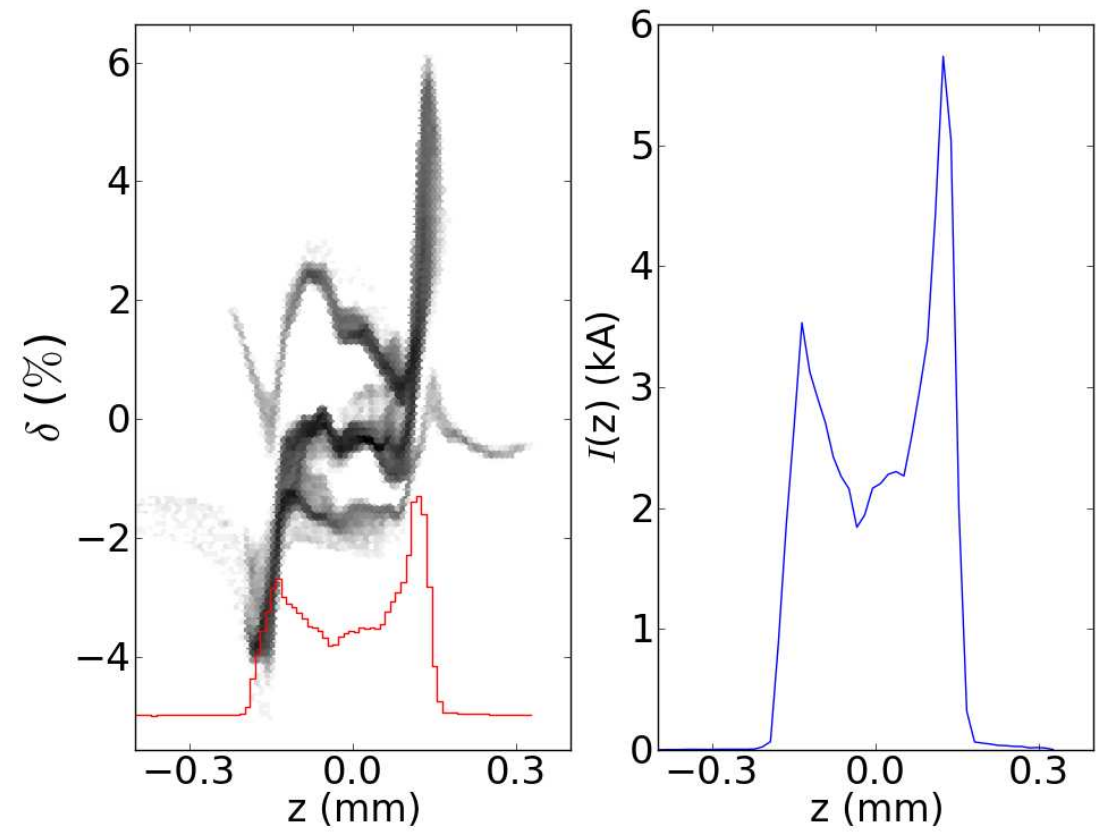

FIG. 26: LPS and associated longitudinal charge distribution (red trace) in arbitrary units (left) and current profile (right) for a 3.2-nC bunch over-compressed with $\mathcal{C}=5.50 \mathrm{~m}^{-1}$, generated in IMPACT-Z. 
[1] M. Church, et al, Proc. of PAC07, 2942 (2007).

[2] J. Leibfritz, et. al, Proc. of PAC11, MOP009 (2011).

[3] C.R. Prokop, et. al, FERMILAB-TM-2516-APC (2011).

[4] P. Piot, et al., Proc. of IPAC10, THPD020 (2010).

[5] T. Smith, in Proceedings of the 1984 Linear Accelerator Conference (LINAC'84), SLAC report 303, 421 (1986).

[6] D. H. Dowell, T. D. Hayward, and A. M. Vetter, in Proceedings of the 1995 Particle Acceleration Conference, Dallas (IEEE, Piscataway, 1996), p. 992.

[7] K. Flöttmann, T. Limberg, Ph. Piot, DESY Report No. TESLA-FEL 2001-06 (2001).

[8] I. Zagorodnov and M. Dohlus, Phys. Rev. ST Accel. Beams 14, 014403 (2011).

[9] E. L. Saldin, et. al, Nucl. Instrum. Meth. in Physics Research A 398 p. 373-394 (1997).

[10] M. Borland, Phys. Rev. ST Accel. Beams 4, 0707012001.

[11] C. R. Prokop, et al., Proc. of PAC2011, p. 1561 (2011).

[12] M. Borland, Advanced Photon Source LS-287, September 2000 (unpublished).

[13] J. Qiang, et al., J. Comp. Phys. 163, p. 434 (2000).

[14] M. D. Dohlus et al., Proc. of the 2004 FEL Conference, p. 18-21 (2004).

[15] M. Dohlus, TESLA-FEL-2003-05.

[16] A. Chao, "Physics of Collective Beam Instabilities in High Energy Accelerators", Wiley \& Sons, Inc. (1993).

[17] M. Dohlus, T. Limberg, in Proc. of PAC05, Knoxville, Tennessee, 1015-1017 (2005).

[18] B.J.Claessens, et al., Phys. Rev. Lett. 95164801 (2005).

[19] R. Brinkmann, Y. Derbenev, and K. Flöttmann, Phys. Rev. ST Accel. Beams 4, 053501 (2001).

[20] K.-J. Kim, Phys. Rev. ST Accel. Beams 6, 104002 (2003).

[21] P. Piot, Y.-E Sun, K.-J. Kim, Phys. Rev. ST Accel. Beams 9, 031001 (2006).

[22] K. L. F. Bane, P. Chen, P. B. Wilson, "On collinear wakefield acceleration", SLAC-PUB-3662 (1985).

[23] P. Piot, C. Behrens, C. Gerth, M. Dohlus, F. Lemery, D. Mihalcea, P. Stoltz, M. Vogt, Phys. Rev. Lett. 108, 034801 (2012). 
[24] F. Lemery, D. Mihalcea, and P. Piot, Proceedings of the 2011 International Particle Accelerator Conference, San Sebastiàn Spain (IPAC11), 2781 (2011).

[25] P. Chen, J.M. Dawson, Robert W. Huff, T. Katsouleas, Phys. Rev. Lett. 54, 693 (1985).

[26] M. Rosing, and W. Gai, Phys. Rev. D 42, 1829 (1990). 